\title{
Optimal discretizations in adaptive finite element electromagnetics
}

\author{
S. McFee ${ }^{* \dagger}$ and D. Giannacopoulos ${ }^{\ddagger}$ \\ Computational Analysis and Design Laboratory, Department of Electrical \& Computer Engineering, \\ McGill University, Montreal, PQ, H3A 2A7, Canada
}

\begin{abstract}
SUMMARY
One of the primary objectives of adaptive finite element analysis research is to determine how to effectively discretize a problem in order to obtain a sufficiently accurate solution efficiently. Therefore, the characterization of optimal finite element solution properties could have significant implications on the development of improved adaptive solver technologies. Ultimately, the analysis of optimally discretized systems, in order to learn about ideal solution characteristics, can lead to the design of better feedback refinement criteria for guiding practical adaptive solvers towards optimal solutions efficiently and reliably. A theoretical framework for the qualitative and numerical study of optimal finite element solutions to differential equations of macroscopic electromagnetics is presented in this study for one- , two- and three-dimensional systems. The formulation is based on variational aspects of optimal discretizations for Helmholtz systems that are closely related to the underlying stationarity principle used in computing finite element solutions to continuum problems. In addition, the theory is adequately general and appropriate for the study of a range of electromagnetics problems including static and time-harmonic phenomena. Moreover, finite element discretizations with arbitrary distributions of element sizes and degrees of approximating functions are assumed, so that the implications of the theory for practical $h-, p$-, $h p$ - and $r$-type finite element adaption in multidimensional analyses may be examined. Copyright (C) 2001 John Wiley \& Sons, Ltd.
\end{abstract}

KEY WORDS: finite element; adaptive methods; electromagnetics; optimal discretizations

\section{INTRODUCTION}

Currently, finite element analysis (FEA) is widely used in computational electromagnetic design and research-typically, FEA tools are used to numerically simulate and evaluate the performance of a new device design before building a prototype [1-16]. In recent years,

\footnotetext{
*Correspondence to: Steve McFee, Department of Electrical and Computer Engineering, McGill University, 3480

University Street, Montreal, PQ, H3A 2A7, Canada

$\dagger$ E-mail: mcfee@ece.mcgill.ca

¥E-mail: dennisg@ece.mcgill.ca

Contract/grant sponsor: Natural Sciences and Engineering Research Council of Canada
}

Copyright (c) 2001 John Wiley \& Sons, Ltd.

Received 16 March 2000

Revised 29 January 2001 
the use of adaptive finite element methods (AFEMs) has received considerable attention for solving problems more efficiently than standard FEMs [17]. In general, finite element solutions are approximate [18]; and their accuracy depends on the number of free parameters used to mathematically model the problem, and on how effectively those parameters, or mathematical degrees of freedom (DOF), are distributed throughout the problem space. In addition, the cost of solving a finite element problem grows with the number of DOF used in the discretization. Consequently, the most efficient distributions of DOF for FEA are those that yield sufficiently accurate solutions using the fewest free parameters. Today, one practical way to achieve this objective is by using adaptive solution strategies which are capable of intelligently evolving and improving efficient distributions of DOF over the problem domain, by establishing error distributions, and then adjusting or adding DOF to the discretization to correct them [19-21].

Standard FEMs increase the number of DOF throughout the problem domain in a uniform fashion by augmenting the existing level of discretization in each subregion (element) equally. Generally, this can result in an inefficient solution process, since most electromagnetics field solutions will not conform to the initial distribution of DOF, and uniform refinements do not allow for this distribution to be changed. However, by augmenting the DOF in the regions of higher solution error only, it is often possible to make significant improvements in the global accuracy of the finite element solution for the least additional computational cost $[19,20]$.

Presently, four basic types of adaption models are under study: $h$-type, $p$-type, $h p$-type and $r$-type. Essentially, these models differ only in the methods used to update the finite element discretization within the adaptive feedback loop [20]:

A. Generate initial discretization.

Repeat:

B. Solve finite element problem.

C. Evaluate solution accuracy; if adequate STOP.

D. Identify regions of inadequate discretization.

E. Update finite element discretization.

Until STOP.

Simply stated, $h$-type adaption models add new elements to the mesh; $p$-type models increase the degree of approximation over pre-existing elements in the mesh; $h p$-type models employ a combination of both procedures; and $r$-type models reposition element vertices in the mesh to improve the solution accuracy. All four adaption models are considered in this work.

Today, many realistic problems require a large number of DOF to compute their solutions with sufficient accuracy; and, the efficient discretization of the physical problem has become an essential requirement of modem finite element packages. These practical requisites have led to an increasingly strong need and demand for advanced adaptive solver technologies for electromagnetics FEA. Currently, the research and development of optimized AFEMs which are effective, reliable and versatile enough for general application in electromagnetics analysis and design, is a critical component of the state-of-the-art in FEA research. The purpose of the present contribution is to advance this frontier, through the development of practical adaptive refinement criteria, which are able to effectively reproduce the main modelling characteristics of optimal discretizations, without the expense of solving the optimal discretization problem. 


\subsection{Technical constraints}

Generally, electromagnetic field problems can be formulated using the operator equation:

$$
\mathscr{L} u=g
$$

where $\mathscr{L}$ is a linear operator that can be chosen so that Equation (1) represents any of Maxwell's integral or differential equations of macroscopic electromagnetics, and $u$ and $g$ are symbolic representations of corresponding scalar or vector fields [22]. In general, $g$ could represent a prescribed source of electromagnetic energy, and $u$ would give the unknown electromagnetic field, or auxiliary potential, which satisfies Equations (1) subject to the boundary conditions for the problem under consideration. When solving electromagnetic problems using FEMs, the basic approach is to define a space of admissible approximating functions, say $V_{h}$, from which the closest function to the true solution $u$, say $u_{h}$, is eventually computed. In general, FEMs use a finite number of fixed-position geometric sub-domains (elements), over which interpolation functions are used to approximate the unknown field $u$. Therefore, one restriction placed on $V_{h}$ is the a priori imposed distribution of some of the mathematical DOF used to model the physical problem due to the pre-assigned fixed topology of these elements.

Standard FEA solutions are only optimal under these restrictions, i.e. the pre-assigned element topology. However, if an operational specification of truly optimal approximate solutions, i.e. the approximate solutions that would result if these restrictions were removed, were known a priori, then practical algorithms could be developed and applied to adaptively compute FEA solutions with similar optimal properties, but at a much lower computational cost and complexity than what would be required to resolve the geometrically unconstrained optimization problems. Although AFEMs are well suited to evolving finite element approximations with optimal-discretization solution properties, this can only be achieved through the application of appropriate feedback criteria for guiding the adaption process towards optimal discretizations. Therefore, the identification and verification of an operational characterization of optimal finite element discretizations forms an essential foundational step of the present contribution.

\subsection{Foundational support}

There has been considerable research on optimal discretizations for numerical modelling methods, including FEMs, in mathematics and engineering [19, 20, 23-41]. Some of the preliminary developments that have played roles in this work are described in [23-27, 42, 43]. For example, the characterization of solution properties associated with optimal discretizations for modern FEMs received considerable theoretical and experimental attention in a series of independent works over the 1970s [24-27]. Unfortunately, this research was largely limited to specialized, low-order, 1-D systems; and for the most part, the conclusions are unsuitable or insufficient for practical use. Some of the most prominent and enduring contributions to emerge are based on the so-called equidistribution principle (EP) [28-30, 32-39, 41]. One of the most general and powerful formulations in this area introduced the concept of the grading function [30]. By this approach, optimal discretizations can be iteratively generated by moving element vertices until a specified grading function changes by the constant amount $1 / N$ over each element in a mesh comprised of $N$ elements. The grading function method of [30] was also investigated in numerous other publications, including [28, 29, 32-39, 41]. Unfortunately, these approaches require explicit knowledge of the exact solution, $u$, in order 
to build the grading function. Therefore, while this method may be suitable for interpolation, it is not sufficient for finite element approximation problems, where the exact field solution is not available in advance. More recently, a number of extended variations of the grading function approach, designed for 2-D, 3-D, and even approximation problems, were reported in References [36, 38-40]. Overall, the most promising of these contributions were subsequently found to be potentially unreliable, or at best inconclusive, for practical finite element applications [44]. Finally, two other EP methods were proposed in References [29, 41]. The first represents a heuristic approach defined for the residual of the governing partial differential equation; the second is based on equidistributing the energy norm of the error. Unfortunately, both of these EP approaches were also determined to be poorly suited to the problem at hand. Overall, and still consistent with the findings of Reference [33], the current body of published knowledge on optimal discretizations is insufficient to provide a reliable operational characterization of optimal finite element discretizations for electromagnetics analysis. A fully expanded review and detailed discussion of the abridged survey presented above is provided in Reference [44]

\subsection{Objectives}

The main purpose of this contribution is to develop practical adaptive refinement criteria which are sufficient to define AFEMs that are able to effectively reproduce the dominant and essential modelling characteristics of optimal finite element discretizations, without incurring the prohibitive costs and complexities of solving for these associated optimal discretizations. As clarified by the current state-of-the-art, an essential prerequisite objective of this work is to identify and validate a foundational operational characterization of optimal discretizations for electromagnetic analysis. This present contribution has been divided into three steps, in order to complete these two goals. First, a mathematical formulation for computing optimal discretizations, which is sufficiently operational to admit an adaptive refinement criterion, is developed from a theoretical basis. Second, the validity and generality of the formulation is confirmed through comprehensive independent testing. Finally, the ability of the refinement criteria to effectively and efficiently reproduce the dominant performance results associated with optimal discretizations, in practical AFEA applications, is investigated and established computationally, and then rated in comparison with the current state-of-the-art.

\section{FORMULATIONS FOR OPTIMAL FINITE ELEMENT SOLUTIONS}

The purpose of this section is two-fold: first, to develop a strongly founded operational formulation for computing optimal finite element discretizations; second, to define a family of refinement criteria with the potential to reproduce the dominant FEA performance results associated with optimal discretizations, within a practical AFEA environment.

The first objective is to assemble a collection of non-linear systems of equations that can be used to simultaneously compute both optimal field solution values and optimal geometric discretization parameters, for finite element formulations of 1-D, 2-D and 3-D scalar boundary value problems in macroscopic electromagnetics. This mathematical formulation is derived, based on well-established variational principles, to be sufficiently general to cover a range of electromagnetic problems including static and time-harmonic phenomena, and finite element 
discretizations entailing arbitrary distributions of element sizes and degrees of approximating functions. A variational method is preferred because it facilitates independent verification and validation through both perturbation testing and solution space searches. Further, variational schemes are intrinsically operational, and readily admit related refinement criteria. Although several different choices exist for the shape of the elements to be used, the typical selection for electromagnetics is the simplex [5,45]. For example, line segments, triangles and tetrahedra are usually used in 1-D, 2-D and 3-D applications, respectively [22]. Simplex elements are used exclusively in this work, however the mathematical formulation is not restricted to any specific choices of approximating functions. Ultimately, the matrix equations corresponding to this formulation will be solved, using standard optimization methods, in order to compute truly optimal finite element solutions for a set of benchmark electromagnetic systems. These solutions will be of the highest accuracy possible for the level of problem refinement specified, for the variational approach used, because the variational principle underlying the problem formulation is itself used as the basis for deriving the optimization equations [44].

The second objective of this section is to specify a template mathematical definition for the potential AFEA refinement criteria associated with the proposed variational formulation.

\subsection{Abstract variational problem}

The usual variational approach used to solve electromagnetic problems with FEMs is to reformulate the continuum field problem defined by Maxwell's equations using a variational principle, to generate a system of algebraic equations which represent a discretized problem, whose solution approximates that of the original continuum problem [22]. The formulation developed here is based on the variational principle of determining the function $u(x, y, z)^{\ddagger}$ which renders the functional $F(u)$ stationary, where

$$
F(u)=\frac{1}{2}\langle\mathscr{L} u, u\rangle-\langle u, g\rangle
$$

The notation $\langle\cdot, \cdot\rangle$ denotes the symmetric product defined over the problem region $\Omega$ as

$$
\langle\phi, \psi\rangle=\int_{\Omega} \phi \psi \mathrm{d} \Omega
$$

where $\phi$ and $\psi$ are real or complex valued scalar functions. ${ }^{\S}$ Assuming that $\mathscr{L}$, subject to its boundary conditions, is a self-adjoint complex operator, i.e.

$$
\langle\mathscr{L} \phi, \psi\rangle=\langle\phi, \mathscr{L} \psi\rangle
$$

then it can be shown [7] that the functional $F(u)$ in Equation (2) will be stationary about a given function $u$ if the following equation is satisfied by $u(x, y, z)$ :

$$
\mathscr{L} u-g=0
$$

\footnotetext{
$¥$ In the discussion above, it is assumed that only admissible functions, i.e. functions which comply with the boundary conditions and continuity requirements of the given electromagnetics problem, and that are sufficiently differentiable to the degree required to evaluate the functional $F$ in Equation (2), are considered for determining the stationary point of the functional $F$.

$\S$ The variational principle described in this section is also applicable to vector problems, for which the inner product is defined as $\langle\mathbf{a}, \mathbf{b}\rangle=\int_{\Omega} \mathbf{a} \cdot \mathbf{b} \mathrm{d} \Omega$. However, only problems that can be expressed in terms of scalar unknowns will be considered in this work.
} 
Therefore, finding the function $u(x, y, z)$ that renders the functional $F(u)$ stationary is equivalent to solving the original electromagnetics problem. Further, the function $u(x, y, z)$ for which $F(u)$ is stationary may be determined uniquely since it must satisfy Maxwell's equations subject to the specific boundary conditions associated with the original electromagnetics problem [46].

In finite element applications, the functional $F$ is uniquely defined by the values of a finite set of parameters associated with the approximating functions used to model the solution over the discretized domain. Therefore, the stationarity requirement amounts to finding the stationary point of $F$ with respect to variations in these parameters, which may be achieved by the usual methods of differential calculus [47]. One important consequence of the fact that this stationarity principle is appropriate for the solution of finite element problems formulated using the above variational approach, is that such formulations can be used to define effective AFEA methods for optimizing finite element discretizations [19].

\subsection{Generalized functional for electromagnetic systems}

A generalized functional is developed for the variational finite element formulation of a range of 1-D, 2-D and 3-D scalar boundary value problems in macroscopic electromagnetics. The approach taken is to begin with a sufficiently general differential equation, together with boundary conditions, and apply the variational principle described in Section 2.1 to derive the corresponding functional. One important merit of this approach is its generality: the formulation is valid for a generalized electromagnetics differential equation; and thereby models problems in Laplace's equation, Poisson's equation, the Helmholtz equation, and a diffusion equation, by simply dropping terms from the final result.

Consider the general, second-order, scalar, partial differential equation:

$$
\nabla \cdot(p \nabla u)+\left(k^{2}+D\right) u=g
$$

in the enclosed region $\Omega$ bounded by the surface $S$, with boundary conditions:

$$
\left.u\right|_{S_{\mathrm{d}}}=u_{\mathrm{d}}
$$

and

$$
\left.\frac{\partial u}{\partial n}\right|_{S_{\mathrm{n}}}=0
$$

The symbols appearing in Equations (6) and (7) have the following meaning:

$u$ electromagnetic field unknown to be solved for

$p$ material-related parameter equal to $\varepsilon, \mu^{-1}$, or 1

$\varepsilon \quad$ permittivity

$\mu$ permeability

$k$ wave number equal to $\omega \sqrt{\mu \varepsilon}$ 
$\omega$ frequency in radians/s

$D$ parameter equal to $-\mathrm{j} \omega \sigma \xi$

$\mathrm{j}$ equal to $\sqrt{-1}$

$\sigma$ conductivity

$\xi \quad$ equal to $\mu, 1$, or 0

$g$ source function

$S_{\mathrm{d}} \quad$ Dirichlet surfaces

$S_{\mathrm{n}} \quad$ Neumann surfaces

The Dirichlet surfaces are those parts of the problem domain boundary on which the solution $u$ must take on a prescribed value $u_{\mathrm{d}}$. Similarly, on Neumann surfaces the normal component of the solution gradient, $\partial u / \partial n$, must take on a prescribed value. The surface bounding the entire problem region $\Omega$, is comprised of the union of the Dirichlet and Neumann surfaces, i.e. $S=S_{\mathrm{d}}+S_{\mathrm{n}}$.

The differential equation (6) may be reformulated as the operator equation (1), if $\mathscr{L}$ is defined as

$$
\mathscr{L}=\nabla \cdot(p \nabla)+\left(k^{2}+D\right)
$$

However, due to the inhomogeneous Dirichlet boundary condition (7), this operator is not self-adjoint for the unknown function $u$. Nevertheless, $\mathscr{L}$ is self-adjoint for the related function $u_{o}$ :

$$
u_{o}=u-\phi
$$

where $\phi$ is any function that satisfies the given inhomogeneous boundary condition. Therefore, if $u$ is substituted by $u_{o}+\phi$ in Equation (1), then the variational principle described earlier may be applied to the equation for $u_{o}$. The corresponding functional, when expanded in terms of Equation (9), may be written as

$$
F(u)=\frac{1}{2} \int_{\Omega}\left\{p \nabla u \cdot \nabla u-\left(k^{2}+D\right) u^{2}+2 g u\right\} \mathrm{d} \Omega
$$

after some simplification, as explained in Reference [44]. Finally, the true solution to Equation (6) over the problem domain $\Omega$, subject to the boundary conditions in Equation (7), is the admissible function $u$ for which the above functional $F$ is stationary.

\subsection{Non-linear system formulation for the finite element equations}

Discretized forms of Equation (10) may be used to compute unique finite element solutions to electromagnetics problems cast in terms of Equations (6) and (7). The approach is to develop a non-linear system of optimization equations from a variational formulation, which can be solved using standard optimization methods to determine optimal values for the solution unknowns. The stationarity condition of the variational principle presented earlier is used as the basis for deriving these optimization equations.

The dependence of the functional $F$ on the solution unknowns may be expressed as

$$
F=F(\mathbf{u}(\mathbf{x}), \mathbf{x})
$$


where the field solution is represented by the vector of field solution parameters $\mathbf{u}$, while $\mathbf{x}$ represents the vector of the unconstrained geometric discretization parameters for the problem. The dependence of the field solution parameters on the discretization parameters is expressed by

$$
\mathbf{u}=\mathbf{u}(\mathbf{x})
$$

Consequently, stationarity of the functional $F$ with respect to variations in $\mathbf{u}$, with the geometric discretization held fixed, is achieved by satisfying

$$
\left.\frac{\partial F}{\partial \mathbf{u}}\right|_{\mathbf{x}}=0
$$

which is equivalent to the usual variational finite element formulation for fixed-position geometric sub-domains. For finite element formulations in which the geometric discretization can also vary, stationarity of the functional with respect to variations in the geometric discretization implies

$$
\frac{\partial F}{\partial \mathbf{x}}=\left.\frac{\partial F}{\partial \mathbf{u}}\right|_{\mathbf{x}} \frac{\partial \mathbf{u}}{\partial \mathbf{x}}+\left.\frac{\partial F}{\partial \mathbf{x}}\right|_{\mathbf{u}}=0
$$

Therefore, based on Equation (13) it follows that

$$
\left.\frac{\partial F}{\partial \mathbf{x}}\right|_{\mathbf{u}}=0
$$

represents the stationarity requirements for the functional $F$ with respect to variations in the geometric discretization of a problem.

The optimization equations required to simultaneously compute optimal field solution values along with optimal geometric discretization parameters for finite element formulations, i.e. Equations (13) and (15) may be re-expressed as the non-linear system:

$$
\mathbf{F}(\mathbf{U})=\mathbf{0}
$$

where the elements of vector $\mathbf{F}$ represent stationarity requirement expressions of the functional $F$ with respect to the elements of the unknown vector $\mathbf{U}=\left[\mathbf{u}^{\mathrm{T}}, \mathbf{x}^{\mathrm{T}}\right]$. For example, in a problem where $\mathscr{N}$ is the number of unknowns to be optimized:

$$
\begin{aligned}
\mathbf{F} & =\left[\mathscr{F}_{1}, \mathscr{F}_{2}, \ldots, \mathscr{F}_{\mathcal{N}}\right]^{\mathrm{T}} \\
\mathbf{U} & =\left[\mathscr{U}_{1}, \mathscr{U}_{2}, \ldots, \mathscr{U}_{\mathcal{N}}\right]^{\mathrm{T}}
\end{aligned}
$$

and

$$
\mathscr{F}_{i}=\frac{\partial F}{\partial \mathscr{U}_{i}}
$$



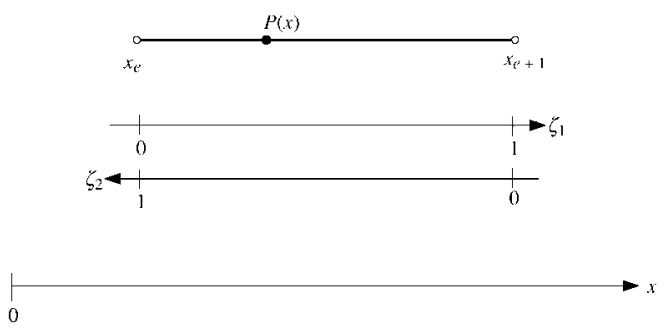

Figure 1. Simplex co-ordinates for a 1-D finite element.

Thus, the non-linear system (16) will have the form

$$
\begin{gathered}
\mathscr{F}_{1}\left(\mathscr{U}_{1}, \mathscr{U}_{2}, \ldots, \mathscr{U}_{N}\right)=0 \\
\mathscr{F}_{2}\left(\mathscr{U}_{1}, \mathscr{U}_{2}, \ldots, \mathscr{U}_{N}\right)=0 \\
\vdots \\
\mathscr{F}_{N}\left(\mathscr{U}_{1}, \mathscr{U}_{2}, \ldots, \mathscr{U}_{N}\right)=0
\end{gathered}
$$

and may be solved using standard optimization methods. The solution $\mathbf{U}$ of (20) is the characterization of the best approximation, from the space of admissible approximations, to the true solution $u$ of Equation (6).

\subsection{Finite element optimization equations}

The purpose of this section is to develop discretized forms of functional (10), and the finite element optimization equations (20). The discretized functionals are derived using conventional approaches detailed in standard references; only the key steps and results are mentioned here. However, significant details that differ from the usual treatments, or that are key to the derivation of the finite element optimization equations, are considered in greater depth. The simpler 1-D results are developed first, to clearly communicate the essential aspects and potential limitations of the formulations; then the more demanding multi-dimensional versions are derived along the same lines.

2.4.1. 1-D systems Electromagnetic systems which possess appropriate geometric symmetry may be analyzed using 1-D finite element formulations. Consider the 1-D element with vertex positions $x_{e}$ and $x_{e+1}$, such that $x_{e+1}>x_{e}$, as shown in Figure 1. The location of a point $P(x)$ within the element may be expressed in terms of the 1-D simplex co-ordinates, $\zeta_{1}$ and $\zeta_{2}$, defined by

$$
\zeta_{1}=\frac{x-x_{e}}{x_{e+1}-x_{e}}, \quad \zeta_{2}=\frac{x_{e+1}-x}{x_{e+1}-x_{e}}, \quad \text { and } \quad \zeta_{1}+\zeta_{2}=1
$$

The purely local nature of simplex co-ordinates permits certain parts of the finite element formulation to be developed independent of the global co-ordinate system. Thereby, results developed for one element are equally applicable to all other elements, by co-ordinate 
transformations. Further, although only one co-ordinate is independent, various quantities arising in certain stages of the formulation are more conveniently expressed in terms of both $\zeta_{1}$ and $\zeta_{2}$.

The field solution $u$ to the differential equation (6) may be approximated, over an individual 1-D element $e$, using a linear combination of basis functions $\alpha_{i}\left(\zeta_{1}, \zeta_{2}\right)$ :

$$
u \approx \sum_{i=0}^{n_{e}} U_{i} \alpha_{i}\left(\zeta_{1}, \zeta_{2}\right)
$$

where the $U_{i}$ are real or complex, constant coefficients, that represent the $n_{e}+1$ field solution unknowns associated with the element. The basis functions in Equation (22) are left general for the moment, however, explicit forms are incorporated later in this work. Substituting the approximation for $u$ into functional (10) yields

$$
F^{(e)}=\frac{1}{2} \sum_{i=0}^{n_{e}} \sum_{j=0}^{n_{e}} U_{i} U_{j} \int_{\Omega_{e}}\left\{p \nabla \alpha_{i} \cdot \nabla \alpha_{j}-\left(k^{2}+D\right) \alpha_{i} \alpha_{j}\right\} \mathrm{d} \Omega+\sum_{i=0}^{n_{e}} U_{i} \int_{\Omega_{e}} g \alpha_{i} \mathrm{~d} \Omega
$$

where $\Omega_{e}$ represents the portion of the problem domain associated with the element $e$, and $F^{(e)}$ represents the corresponding portion of the functional.

For 1-D systems where $x$ represents the independent co-ordinate variable for the solution $u(x)$, it may be confirmed that

$$
\nabla \alpha_{i} \cdot \nabla \alpha_{j}=\frac{\partial \alpha_{i}}{\partial x} \frac{\partial \alpha_{j}}{\partial x}=\frac{1}{h_{e}^{2}} \frac{\partial \alpha_{i}}{\partial \zeta_{1}} \frac{\partial \alpha_{j}}{\partial \zeta_{1}}
$$

by noting

$$
\frac{\partial \alpha_{i}}{\partial x}=\frac{\partial \alpha_{i}}{\partial \zeta_{1}} \frac{\partial \zeta_{1}}{\partial x}+\frac{\partial \alpha_{i}}{\partial \zeta_{2}} \frac{\partial \zeta_{2}}{\partial x} \quad \text { and } \quad \frac{\partial \zeta_{1}}{\partial x}=\frac{1}{h_{e}}, \frac{\partial \zeta_{2}}{\partial x}=-\frac{1}{h_{e}}
$$

where $h_{e}$ is the length of the element, i.e.

$$
h_{e}=x_{e+1}-x_{e}
$$

Further, it may be confirmed that:

$$
\mathrm{d} \Omega=x^{r} \mathrm{~d} x=\left(\zeta_{1} h_{e}+x_{e}\right)^{r} h_{e} \mathrm{~d} \zeta_{1} \quad(r=0,1,2)
$$

given the 1-D co-ordinate transformation:

$$
\mathrm{d} \zeta_{1}=\frac{\partial \zeta_{1}}{\partial x} \mathrm{~d} x=\frac{1}{h_{e}} \mathrm{~d} x
$$

and in view of (as determined by Equations (21) and (26))

$$
x=\zeta_{1} h_{e}+x_{e}
$$


Therefore, upon substituting Equations (24) and (27) into Equation (23), the general form of the discretized functional over a typical element $e$ becomes

$$
\begin{aligned}
F^{(e)}= & \frac{1}{2} \sum_{i=0}^{n_{e}} \sum_{j=0}^{n_{e}} U_{i} U_{j} \int_{\zeta_{1}=0}^{1} \frac{p_{e}}{h_{e}^{2}} \frac{\partial \alpha_{i}}{\partial \zeta_{1}} \frac{\partial \alpha_{j}}{\partial \zeta_{1}}\left[\zeta_{1} h_{e}+x_{e}\right]^{r} h_{e} \mathrm{~d} \zeta_{1} \\
& -\frac{1}{2} \sum_{i=0}^{n_{e}} \sum_{j=0}^{n_{e}} U_{i} U_{j} \int_{\zeta_{1}=0}^{1}\left(k_{e}^{2}+D_{e}\right) \alpha_{i} \alpha_{j}\left[\zeta_{1} h_{e}+x_{e}\right]^{r} h_{e} \mathrm{~d} \zeta_{1} \\
& +\sum_{i=0}^{n_{e}} U_{i} \int_{\zeta_{1}=0}^{1} g_{e} \alpha_{i}\left[\zeta_{1} h_{e}+x_{e}\right]^{r} h_{e} \mathrm{~d} \zeta_{1}
\end{aligned}
$$

where $p_{e}, k_{e}, D_{e}$, and $g_{e}$ represent the specific forms of $p, k, D$, and $g$ in $\Omega_{e}$, respectively. (Note: these quantities are assumed to be expressed purely in terms of the single simplex coordinate $\zeta_{1}$.) Finally, the dependency of the functional on the underlying reference co-ordinate system is determined by the value of the parameter $r$ :

$r=0$ corresponds to a Cartesian co-ordinate system: $(x, y, z)$;

$r=1$ corresponds to a circular cylindrical co-ordinate system: $(\rho, \phi, z)$; and,

$r=2$ corresponds to a spherical co-ordinate system: $(R, \theta, \phi)$.

Although the functional $F^{(e)}$ corresponds to a 1-D formulation, the contribution from each element in the three-dimensional volume associated with the physical problem must be included in $F^{(e)}$ in order to correctly apply the variational principle. Hence, the Jacobian $\left(\zeta_{1} h_{e}+x_{e}\right)^{r} h_{e}$ in Equation (30) corresponds to transformations from the differential elements of volume associated with the three common co-ordinate systems, as defined by the value of $r$. However, any factors which do not differ in the unit volume from one element to the next, for a particular co-ordinate system, have not been included in the expression for the elemental functional $F^{(e)}$ in Equation (30). Specifically, when considering 1-D problems defined with respect to the three reference co-ordinate systems, the independent co-ordinate variable is taken to be either $x, \rho$, or $R$ in each case, respectively. However, the independent co-ordinate variable in all three cases is consistently represented by the symbol $x$. Thus, the volume corresponding to each element in a given 1-D discretization is determined, to within a common multiplicative factor, by considering the differential volume element defined in Equation (27).

The functional $F^{(e)}$ defined in Equation (30) may be expressed more concisely by defining

$$
\begin{aligned}
& V_{i j}^{(e)}=\int_{\zeta_{1}=0}^{1} \frac{p_{e}}{h_{e}^{2}} \frac{\partial \alpha_{i}}{\partial \zeta_{1}} \frac{\partial \alpha_{j}}{\partial \zeta_{1}}\left[\zeta_{1} h_{e}+x_{e}\right]^{r} h_{e} \mathrm{~d} \zeta_{1} \\
& B_{i j}^{(e)}=\int_{\zeta_{1}=0}^{1}\left(k_{e}^{2}+D_{e}\right) \alpha_{i} \alpha_{j}\left[\zeta_{1} h_{e}+x_{e}\right]^{r} h_{e} \mathrm{~d} \zeta_{1}
\end{aligned}
$$

and

$$
f_{i}^{(e)}=\int_{\zeta_{1}=0}^{1} g_{e} \alpha_{i}\left[\zeta_{1} h_{e}+x_{e}\right]^{r} h_{e} \mathrm{~d} \zeta_{1}
$$




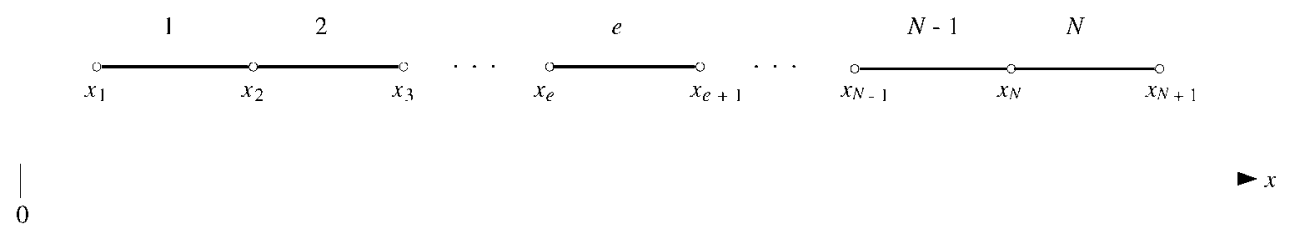

Figure 2. A 1-D finite element mesh of $N$ elements.

Substituting for these integrals in Equation (30) yields

$$
F^{(e)}=\frac{1}{2} \sum_{i=0}^{n_{e}} \sum_{j=0}^{n_{e}} U_{i} U_{j} V_{i j}^{(e)}-\frac{1}{2} \sum_{i=0}^{n_{e}} \sum_{j=0}^{n_{e}} U_{i} U_{j} B_{i j}^{(e)}+\sum_{i=0}^{n_{e}} U_{i} f_{i}^{(e)}
$$

or, expressed in matrix form

$$
F^{(e)}=\frac{1}{2} \mathbf{u}^{(e) \mathrm{T}} \mathbf{V}^{(e)} \mathbf{u}^{(e)}-\frac{1}{2} \mathbf{u}^{(e) \mathrm{T}} \mathbf{B}^{(e)} \mathbf{u}^{(e)}+\mathbf{u}^{(e) \mathrm{T}} \mathbf{f}^{(e)}
$$

where the vector $\mathbf{u}^{(e)}$ consists of the field solution unknowns $U_{i}$, for the element $e$, of a 1-D finite element discretization comprised of $N$ elements, as shown in Figure 2. The functional for the full discretization is given by the sum of the elemental contributions:

$$
F=\sum_{e=1}^{N} F^{(e)}
$$

The optimization equations for the field solution parameters $\mathbf{u}^{(e)}$ are derived from the stationarity requirements

$$
\frac{\partial F^{(e)}}{\partial U_{i}}=0 \quad\left(i=0,1, \ldots, n_{e}\right)
$$

or equivalently

$$
\frac{\partial F^{(e)}}{\partial \mathbf{u}^{(e)}}=\mathbf{0}
$$

Applying the identity

$$
\frac{\partial}{\partial \mathbf{u}}\left(\mathbf{u}^{\mathrm{T}} \mathbf{w}\right)=\left(\frac{\partial \mathbf{u}^{\mathrm{T}}}{\partial \mathbf{u}}\right) \mathbf{w}+\left(\frac{\partial \mathbf{w}^{\mathrm{T}}}{\partial \mathbf{u}}\right) \mathbf{u}=\mathbf{w}+\left(\frac{\partial \mathbf{w}^{\mathrm{T}}}{\partial \mathbf{u}}\right) \mathbf{u}
$$

in view of the symmetry of the matrices $\mathbf{V}^{(e)}$ and $\mathbf{B}^{(e)}$, Equation (36) yields

$$
\mathbf{V}^{(e)} \mathbf{u}^{(e)}-\mathbf{B}^{(e)} \mathbf{u}^{(e)}+\mathbf{f}^{(e)}=\mathbf{0}
$$

Therefore, considering all the elements in a discretization yields the set of equations:

$$
\mathbf{V}^{(e)} \mathbf{u}^{(e)}-\mathbf{B}^{(e)} \mathbf{u}^{(e)}=-\mathbf{f}^{(e)} \quad(e=1,2, \ldots, N)
$$

which may be solved to optimize the values of the global field solution parameters $\mathbf{u}$. In practical finite element implementations many field solution parameters are common to a 
number of elements. The optimization equations corresponding to such parameters may be consolidated into a single equation by consolidating any field solution parameter common to more than one element into a single unknown (e.g. Reference [22]).

The optimization equations for the geometric discretization parameters may be derived from the stationarity requirements

$$
\frac{\partial F}{\partial x_{e}}=0 \quad(e=2,3, \ldots, N)
$$

where the positions of the end-point vertices, $x_{1}$ and $x_{N+1}$, are constrained to the boundaries of the problem domain. Recalling Equation (34), where $F^{(e)}$ depends on the discretization parameters such that

$$
F^{(e)}=F^{(e)}\left(x_{e}, h_{e}\left(x_{e}, x_{e+1}\right)\right)
$$

implies

$$
F^{(e-1)}=F^{(e-1)}\left(x_{e-1}, h_{e-1}\left(x_{e-1}, x_{e}\right)\right)
$$

and thereby

$$
\frac{\partial F}{\partial x_{e}}=\frac{\partial F^{(e-1)}}{\partial x_{e}}+\frac{\partial F^{(e)}}{\partial x_{e}}
$$

where

$$
\frac{\partial F^{(e-1)}}{\partial x_{e}}=\frac{\partial F^{(e-1)}}{\partial x_{e}}+\frac{\partial F^{(e-1)}}{\partial h_{e-1}} \frac{\partial h_{e-1}}{\partial x_{e}}
$$

However, the first term on the right hand side in Equation (44) is equal to zero since $x_{e}$ does not appear explicitly in $F^{(e-1)}$; and from Equation (26):

$$
\frac{\partial h_{e-1}}{\partial x_{e}}=1
$$

Hence

$$
\frac{\partial F^{(e-1)}}{\partial x_{e}}=\frac{\partial F^{(e-1)}}{\partial h_{e-1}}
$$

Similarly

$$
\frac{\partial F^{(e)}}{\partial x_{e}}=\frac{\partial F^{(e)}}{\partial x_{e}}+\frac{\partial F^{(e)}}{\partial h_{e}} \frac{\partial h_{e}}{\partial x_{e}}
$$

in which, from Equation (26)

$$
\frac{\partial h_{e}}{\partial x_{e}}=-1
$$

and therefore

$$
\frac{\partial F^{(e)}}{\partial x_{e}}=\frac{\partial F^{(e)}}{\partial x_{e}}-\frac{\partial F^{(e)}}{\partial h_{e}}
$$


Finally, substituting Equations (46) and (43) into Equation (43) yields

$$
\frac{\partial F}{\partial x_{e}}=\frac{\partial F^{(e-1)}}{\partial h_{e-1}}+\frac{\partial F^{(e)}}{\partial x_{e}}-\frac{\partial F^{(e)}}{\partial h_{e}}
$$

Thus, the set of optimization equations corresponding to the entire set of geometric discretization parameters for a given problem is defined by

$$
\frac{\partial F^{(e-1)}}{\partial h_{e-1}}+\frac{\partial F^{(e)}}{\partial x_{e}}-\frac{\partial F^{(e)}}{\partial h_{e}}=0 \quad(e=2,3, \ldots, N)
$$

which may also be expressed in matrix form as

$$
\begin{aligned}
& \frac{1}{2}\left(\mathbf{u}^{(e-1) \mathrm{T}} \mathbf{P}^{(e-1)} \mathbf{u}^{(e-1)}+\mathbf{u}^{(e) \mathrm{T}} \mathbf{S}^{(e)} \mathbf{u}^{(e)}\right) \\
& \quad-\frac{1}{2}\left(\mathbf{u}^{(e-1) \mathrm{T}} \mathbf{Q}^{(e-1)} \mathbf{u}^{(e-1)}+\mathbf{u}^{(e) \mathrm{T}} \mathbf{T}^{(e)} \mathbf{u}^{(e)}\right) \\
& \quad+\mathbf{u}^{(e-1) \mathrm{T}} \mathbf{d}^{(e-1)}+\mathbf{u}^{(e) \mathrm{T}} \mathbf{r}^{(e)}=0 \quad(e=2,3, \ldots, N)
\end{aligned}
$$

where the matrices and vectors in the above equation are defined by

$$
\begin{aligned}
P_{i j}^{(e-1)} & =\frac{\partial}{\partial h_{e-1}} V_{i j}^{(e-1)} \\
S_{i j}^{(e)} & =\left[\frac{\partial}{\partial x_{e}}-\frac{\partial}{\partial h_{e}}\right] V_{i j}^{(e)} \\
Q_{i j}^{(e-1)} & =\frac{\partial}{\partial h_{e-1}} B_{i j}^{(e-1)} \\
T_{i j}^{(e)} & =\left[\frac{\partial}{\partial x_{e}}-\frac{\partial}{\partial h_{e}}\right] B_{i j}^{(e)} \\
d_{i}^{(e-1)} & =\frac{\partial}{\partial h_{e-1}} f_{i}^{(e-1)}
\end{aligned}
$$

and

$$
r_{i}^{(e)}=\left[\frac{\partial}{\partial x_{e}}-\frac{\partial}{\partial h_{e}}\right] f_{i}^{(e)}
$$

Solving Equations (39) and (52) simultaneously for the field solution unknowns $\mathbf{u}$, and the discretization parameters $\mathbf{x}$, yields the optimal finite element solution for a given problem within the space of admissible solutions defined by the chosen sets of basis functions. Note that Equations (39) are equivalent to the set of equations which define the field solution unknowns in conventional finite element formulations which have fixed-position elements. Further, note that Equations (52) may not be solved independently of Equations (39) for the discretization 


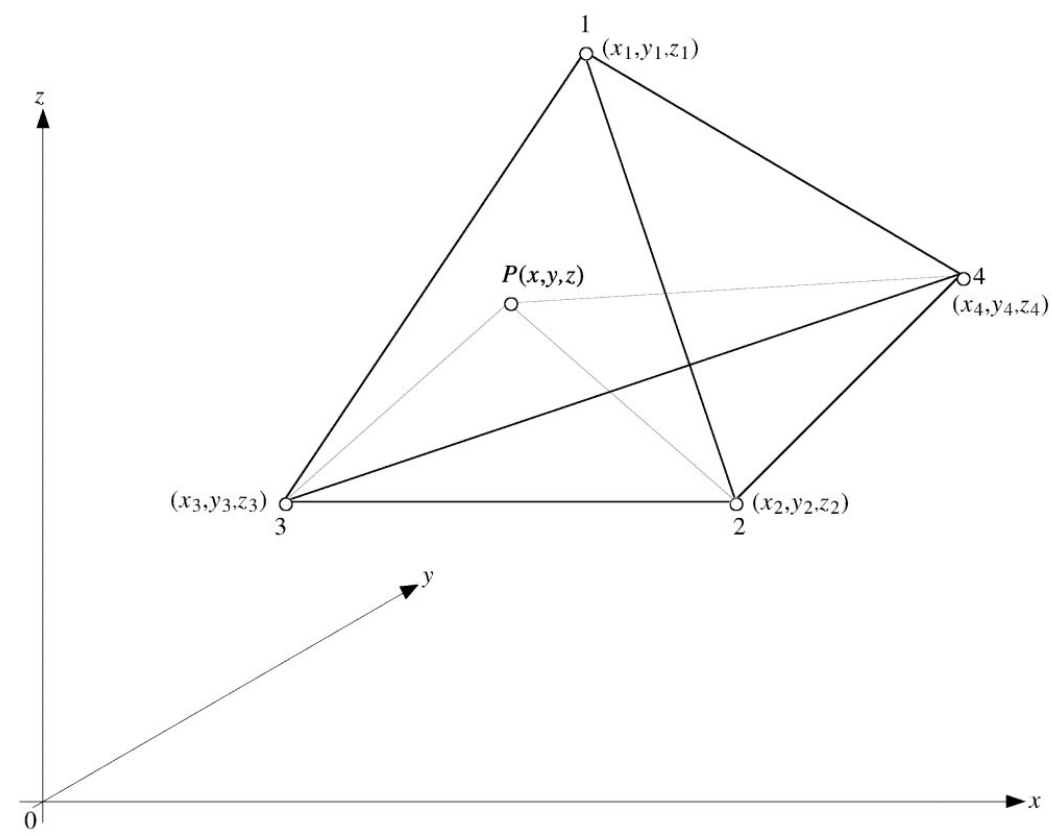

Figure 3. A tetrahedral element and the sub-simplex $P 234$ used in the definition of $\zeta_{1}$.

parameters, since the former have been derived based on the condition of stationarity of the functional with respect to the field solution parameters. In general, stationarity of the functional with respect to the field solution parameters is dependent on the values of the discretization parameters, i.e. the element vertex positions. This dependence is due to the fact that the approximating functions over individual elements are defined by the values of both their associated field solution parameters and the element vertex positions.

2.4.2. 2-D and 3-D systems. Many practical electromagnetic systems do not possess the appropriate symmetry to allow for 1-D treatments, and can only be analysed using 2-D or 3-D formulations. The counterparts of the optimization equations derived for 1-D systems may be developed in a common form for 2-D and 3-D simplex elements. However, while the principal steps of the development are the same, the specifics of their implementation can be substantially different, and in some instances considerably more complex for the 3-D case [44]. Consequently, the more general 3-D case is considered first, followed by a brief explanation for extracting the corresponding 2-D equations.

Consider the tetrahedral element with vertex positions $\left(x_{i}, y_{i}, z_{i}\right), i=1,2,3,4$, as defined in Figure 3. The location of a point $P(x, y, z)$ in the element may be expressed in terms of the volume-based simplex co-ordinates $\zeta_{1}, \zeta_{2}, \zeta_{3}$, and $\zeta_{4}$, which are defined as

$$
\zeta_{1}=\frac{\operatorname{Vol}(P 234)}{\operatorname{Vol}(1234)}, \quad \zeta_{2}=\frac{\operatorname{Vol}(1 P 34)}{\operatorname{Vol}(1234)}, \quad \zeta_{3}=\frac{\operatorname{Vol}(12 P 4)}{\operatorname{Vol}(1234)}, \quad \zeta_{4}=\frac{\operatorname{Vol}(123 P)}{\operatorname{Vol}(1234)}
$$


and satisfy the relationship: $\zeta_{1}+\zeta_{2}+\zeta_{3}+\zeta_{4}=1$. Just as in the 1-D case, the field solution $u$, to the differential equation (6), may be approximated over each tetrahedron in a 3-D discretization, e.g. in element $e$ :

$$
u \approx \sum_{i=0}^{n_{e}} U_{i} \alpha_{i}\left(\zeta_{1}, \zeta_{2}, \zeta_{3}, \zeta_{4}\right)
$$

Here, the $\alpha_{i}\left(\zeta_{1}, \zeta_{2}, \zeta_{3}, \zeta_{4}\right)$ are 3-D basis functions, and the $U_{i}$ are the constant coefficients that represent the $n_{e}+1$ field solution unknowns associated with the element $e$. Similarly, the source function $g$, in Equation (6), may be approximated by

$$
g \approx \sum_{i=0}^{n_{e}} G_{i} \alpha_{i}\left(\zeta_{1}, \zeta_{2}, \zeta_{3}, \zeta_{4}\right)
$$

where the $G_{i}$ are known constant coefficients. Following the 1-D line of derivation, after the approximations for $u$ and $g$ are substituted in functional (10), it can be shown that the general form of the discretized functional over a typical element $e$, may be expressed as [22]

$$
\begin{aligned}
F^{(e)}= & \frac{1}{2} \sum_{i=0}^{n_{e}} \sum_{j=0}^{n_{e}} U_{i} U_{j}\left[\frac{1}{6 \mathscr{V}_{e}} \sum_{m=1}^{4} \sum_{n=1}^{4}\left(b_{m} b_{n}+c_{m} c_{n}+d_{m} d_{n}\right) I_{i j m n}^{(e)}\right] \\
& -\frac{1}{2} \sum_{i=0}^{n_{e}} \sum_{j=0}^{n_{e}} U_{i} U_{j} 6 \mathscr{V}_{e} B_{i j}^{(e)}+\sum_{i=0}^{n_{e}} \sum_{j=0}^{n_{e}} U_{i} G_{j} 6 \mathscr{V}_{e} H_{i j}^{(e)}
\end{aligned}
$$

where $\mathscr{V}_{e}$ is the element volume, $b_{i}, c_{i}$, and $d_{i}$ are defined as follows, based on the tetrahedron vertices specified in Figure 3, with the subscripts progressing modulo 4:

$$
\begin{aligned}
& b_{i}=(-1)^{i}\left|\begin{array}{lll}
1 & y_{i+1} & z_{i+1} \\
1 & y_{i+2} & z_{i+2} \\
1 & y_{i-1} & z_{i-1}
\end{array}\right| \\
& c_{i}=(-1)^{i+1}\left|\begin{array}{ccc}
1 & x_{i+1} & z_{i+1} \\
1 & x_{i+2} & z_{i+2} \\
1 & x_{i-1} & z_{i-1}
\end{array}\right| \\
& d_{i}=(-1)^{i}\left|\begin{array}{lll}
1 & x_{i+1} & y_{i+1} \\
1 & x_{i+2} & y_{i+2} \\
1 & x_{i-1} & y_{i-1}
\end{array}\right|
\end{aligned}
$$

and

$$
\begin{aligned}
I_{i j m n}^{(e)} & =\int_{\zeta_{1}=0}^{1} \int_{\zeta_{2}=0}^{1-\zeta_{1}} \int_{\zeta_{3}=0}^{1-\zeta_{1}-\zeta_{2}} p_{e} \frac{\partial \alpha_{i}}{\partial \zeta_{m}} \frac{\partial \alpha_{j}}{\partial \zeta_{n}} \mathrm{~d} \zeta_{1} \mathrm{~d} \zeta_{2} \mathrm{~d} \zeta_{3} \\
B_{i j}^{(e)} & =\int_{\zeta_{1}=0}^{1} \int_{\zeta_{2}=0}^{1-\zeta_{1}} \int_{\zeta_{3}=0}^{1-\zeta_{1}-\zeta_{2}}\left(k_{e}^{2}+D_{e}\right) \alpha_{i} \alpha_{j} \mathrm{~d} \zeta_{1} \mathrm{~d} \zeta_{2} \mathrm{~d} \zeta_{3}
\end{aligned}
$$




$$
H_{i j}^{(e)}=\int_{\zeta_{1}=0}^{1} \int_{\zeta_{2}=0}^{1-\zeta_{1}} \int_{\zeta_{3}=0}^{1-\zeta_{1}-\zeta_{2}} \alpha_{i} \alpha_{j} \mathrm{~d} \zeta_{1} \mathrm{~d} \zeta_{2} \mathrm{~d} \zeta_{3}
$$

Note that the integrands of Equations (61)-(63) are expressed purely in terms of the three simplex coordinates $\left(\zeta_{1}, \zeta_{2}, \zeta_{3}\right)$. Therefore, $I_{i j m n}^{(e)}, B_{i j}^{(e)}$, and $H_{i j}^{(e)}$ are independent of both the size and shape of the tetrahedral element, i.e. they do not involve the element vertex positions.

As in the 1-D case, the functional corresponding to a typical element $e$, given in Equation (57), also may be expressed in matrix form:

$$
F^{(e)}=\frac{1}{2} \mathbf{u}^{(e) \mathrm{T}} \mathbf{V}^{(e)} \mathbf{u}^{(e)}-\frac{1}{2} \mathbf{u}^{(e) \mathrm{T}}\left(6 \mathscr{V}_{e} \mathbf{B}^{(e)}\right) \mathbf{u}^{(e)}+\mathbf{u}^{(e) \mathrm{T}}\left(6 \mathscr{V}_{e} \mathbf{H}^{(e)}\right) \mathbf{g}^{(e)}
$$

where $\mathbf{u}^{(e)}$ and $\mathbf{g}^{(e)}$ are the element-wise vectors of the field solution and source term parameters, respectively; and the entries of matrix $\mathbf{V}^{(e)}$ are defined by

$$
V_{i j}^{(e)}=\frac{1}{6 \mathscr{V}_{e}} \sum_{m=1}^{4} \sum_{n=1}^{4}\left(b_{m} b_{n}+c_{m} c_{n}+d_{m} d_{n}\right) I_{i j m n}^{(e)}
$$

The functional for the full problem domain, comprising $N$ elements, is given by

$$
F=\sum_{e=1}^{N} F^{(e)}
$$

The optimization equations for the field solution parameters $\mathbf{u}^{(e)}$, associated with a typical element $e$, may be derived from the functional stationarity equations:

$$
\frac{\partial F^{(e)}}{\partial \mathbf{u}^{(e)}}=\mathbf{0}
$$

By the symmetry of the matrices in Equation (64), Equation (67) implies the Equations [22]

$$
\mathbf{V}^{(e)} \mathbf{u}^{(e)}-\left(6 \mathscr{V}_{e} \mathbf{B}^{(e)}\right) \mathbf{u}^{(e)}=-\left(6 \mathscr{V}_{e} \mathbf{H}^{(e)}\right) \mathbf{g}^{(e)} \quad(e=1,2, \ldots, N)
$$

which may be solved for the optimal values of the entire set of field solution parameters $\mathbf{u}$, if the geometric discretization is held fixed. As in the 1-D case, any optimization equations corresponding to field solution unknowns which are common to more than one element, may be consolidated into a single optimization equation.

The optimization equations for the geometric discretization parameters, i.e. the element vertex positions, associated with a tetrahedral element $e$, may be derived by considering the functional stationarity equations defined by

$$
\begin{aligned}
& \frac{\partial F^{(e)}}{\partial x_{l}^{(e)}}=0 \quad(l=1,2,3,4) \\
& \frac{\partial F^{(e)}}{\partial y_{l}^{(e)}}=0 \quad(l=1,2,3,4)
\end{aligned}
$$

and

$$
\frac{\partial F^{(e)}}{\partial z_{l}^{(e)}}=0 \quad(l=1,2,3,4)
$$


where $x_{l}^{(e)}, y_{l}^{(e)}$ and $z_{l}^{(e)}$ represent the $x, y$ and $z$ co-ordinate values of the position of vertex $l(l=1,2,3,4)$ for element $e$, respectively. Since several elements may share a common vertex in a 3-D tetrahedral mesh, it is not generally possible to pre-determine the mesh topology. Therefore, it is necessary to also derive the functional stationarity equations for the discretization parameters in a local manner, i.e. element-wise. As before, the functional stationarity expressions corresponding to a discretization parameter common to more than one element may be consolidated into a single optimization equation.

To derive more explicit forms of Equations (69)-(71), it may be confirmed that the component-wise partial derivatives of $\mathbf{V}^{(e)}$ with respect to $x_{l}^{(e)}$ are given by

$$
\frac{\partial V_{i j}^{(e)}}{\partial x_{l}^{(e)}}=\frac{1}{36 \mathscr{V}_{e}^{2}} \sum_{m=1}^{4} \sum_{n=1}^{4}\left[6 \mathscr{V}_{e}\left(\frac{\partial\left(c_{m} c_{n}\right)}{\partial x_{l}^{(e)}}+\frac{\partial\left(d_{m} d_{n}\right)}{\partial x_{l}^{(e)}}\right)-\left(b_{m} b_{n}+c_{m} c_{n}+d_{m} d_{n}\right) b_{l}\right] I_{i j m n}^{(e)}
$$

which may be obtained from Equation (65) upon noting that

$$
\frac{\partial\left(b_{m} b_{n}\right)}{\partial x_{l}^{(e)}}=0 \quad(m, n, l=1,2,3,4)
$$

and

$$
\frac{\partial\left(6 \mathscr{V}_{e}\right)}{\partial x_{l}^{(e)}}=b_{l} \quad(l=1,2,3,4)
$$

as explained in Reference [44]. In a similar manner, it may be also confirmed that

$$
\frac{\partial V_{i j}^{(e)}}{\partial y_{l}^{(e)}}=\frac{1}{36 \mathscr{V}_{e}^{2}} \sum_{m=1}^{4} \sum_{n=1}^{4}\left[6 \mathscr{V}_{e}\left(\frac{\partial\left(b_{m} b_{n}\right)}{\partial y_{l}^{(e)}}+\frac{\partial\left(d_{m} d_{n}\right)}{\partial y_{l}^{(e)}}\right)-\left(b_{m} b_{n}+c_{m} c_{n}+d_{m} d_{n}\right) c_{l}\right] I_{i j m n}^{(e)}
$$

and

$$
\frac{\partial V_{i j}^{(e)}}{\partial z_{l}^{(e)}}=\frac{1}{36 \mathscr{V}_{e}^{2}} \sum_{m=1}^{4} \sum_{n=1}^{4}\left[6 \mathscr{V}_{e}\left(\frac{\partial\left(b_{m} b_{n}\right)}{\partial z_{l}^{(e)}}+\frac{\partial\left(c_{m} c_{n}\right)}{\partial z_{l}^{(e)}}\right)-\left(b_{m} b_{n}+c_{m} c_{n}+d_{m} d_{n}\right) d_{l}\right] I_{i j m n}^{(e)}
$$

Further, since $\mathbf{u}^{(e)}, \mathbf{g}^{(e)}, \mathbf{B}^{(e)}$, and $\mathbf{H}^{(e)}$ are independent of the geometric discretization parameters, the optimization equations for the geometric discretization parameters associated with a given tetrahedral element $e$, may be expressed as

$$
\begin{array}{cc}
\frac{1}{2} \mathbf{u}^{(e) \mathrm{T}} \mathbf{S}^{(e)} \mathbf{u}^{(e)}-\frac{b_{l}}{2} \mathbf{u}^{(e) \mathrm{T}} \mathbf{B}^{(e)} \mathbf{u}^{(e)}+b_{l} \mathbf{u}^{(e) \mathrm{T}} \mathbf{H}^{(e)} \mathbf{g}^{(e)}=0 & (l=1,2,3,4) \\
\frac{1}{2} \mathbf{u}^{(e) \mathrm{T}} \mathbf{T}^{(e)} \mathbf{u}^{(e)}-\frac{c_{l}}{2} \mathbf{u}^{(e) \mathrm{T}} \mathbf{B}^{(e)} \mathbf{u}^{(e)}+c_{l} \mathbf{u}^{(e) \mathrm{T}} \mathbf{H}^{(e)} \mathbf{g}^{(e)}=0 \quad(l=1,2,3,4)
\end{array}
$$

and

$$
\frac{1}{2} \mathbf{u}^{(e) \mathrm{T}} \mathbf{W}^{(e)} \mathbf{u}^{(e)}-\frac{d_{l}}{2} \mathbf{u}^{(e) \mathrm{T}} \mathbf{B}^{(e)} \mathbf{u}^{(e)}+d_{l} \mathbf{u}^{(e) \mathrm{T}} \mathbf{H}^{(e)} \mathbf{g}^{(e)}=0 \quad(l=1,2,3,4)
$$


Table I. Explicit 3-D forms of $\partial\left(b_{m} b_{n}\right) / \partial y_{l}^{(e)}$ in terms of $b_{i}$ and $\mathscr{Z}_{i j}$ for $m, n, l=1,2,3,4$.

\begin{tabular}{|c|c|c|c|c|}
\hline$(m, n) / l$ & 1 & 2 & 3 & 4 \\
\hline $\begin{array}{l}(1,1) \\
(1,2),(2,1) \\
(1,3),(3,1) \\
(1,4),(4,1) \\
(2,2) \\
(2,3),(3,2) \\
(2,4),(4,2) \\
(3,3) \\
(3,4),(4,3) \\
(4,4)\end{array}$ & $\begin{array}{c}0 \\
b_{1} \mathscr{Z}_{34} \\
b_{1} \mathscr{Z}_{42} \\
b_{1} \mathscr{Z}_{23} \\
2 b_{2} \mathscr{Z}_{34} \\
b_{3} \mathscr{Z}_{34}+b_{2} \mathscr{Z}_{42} \\
b_{4} \mathscr{Z}_{34}+b_{2} \mathscr{Z}_{23} \\
2 b_{3} \mathscr{Z}_{42} \\
b_{4} \mathscr{Z}_{42}+b_{3} \mathscr{Z}_{23} \\
2 b_{4} \mathscr{Z}_{23}\end{array}$ & $\begin{array}{c}2 b_{1} \mathscr{Z}_{43} \\
b_{2} \mathscr{Z}_{43} \\
b_{3} \mathscr{Z}_{43}+b_{1} \mathscr{Z}_{14} \\
b_{4} \mathscr{Z}_{43}+b_{1} \mathscr{Z}_{31} \\
0 \\
b_{2} \mathscr{Z}_{14} \\
b_{2} \mathscr{Z}_{31} \\
2 b_{3} \mathscr{Z}_{14} \\
b_{4} \mathscr{Z}_{14}+b_{3} \mathscr{Z}_{31} \\
2 b_{4} \mathscr{Z}_{31}\end{array}$ & $\begin{array}{c}2 b_{1} \mathscr{Z}_{24} \\
b_{2} \mathscr{Z}_{24}+b_{1} \mathscr{Z}_{41} \\
b_{3} \mathscr{Z}_{24} \\
b_{4} \mathscr{Z}_{24}+b_{1} \mathscr{Z}_{12} \\
2 b_{2} \mathscr{Z}_{41} \\
b_{3} \mathscr{Z}_{41} \\
b_{4} \mathscr{Z}_{41}+b_{2} \mathscr{Z}_{12} \\
0 \\
b_{3} \mathscr{Z}_{12} \\
2 b_{4} \mathscr{Z}_{12}\end{array}$ & $\begin{array}{c}2 b_{1} \mathscr{Z}_{32} \\
b_{2} \mathscr{Z}_{32}+b_{1} \mathscr{Z}_{13} \\
b_{3} \mathscr{Z}_{32}+b_{1} \mathscr{Z}_{21} \\
b_{4} \mathscr{Z}_{32} \\
2 b_{2} \mathscr{Z}_{13} \\
b_{3} \mathscr{Z}_{13}+b_{2} \mathscr{Z}_{21} \\
b_{4} \mathscr{Z}_{13} \\
2 b_{3} \mathscr{Z}_{21} \\
b_{4} \mathscr{Z}_{21} \\
0\end{array}$ \\
\hline
\end{tabular}

where the entries of the matrices $\mathbf{S}^{(e)}, \mathbf{T}^{(e)}$ and $\mathbf{W}^{(e)}$ are defined by

$$
\begin{aligned}
S_{i j}^{(e)} & =\frac{\partial V_{i j}^{(e)}}{\partial x_{l}^{(e)}} \\
T_{i j}^{(e)} & =\frac{\partial V_{i j}^{(e)}}{\partial y_{l}^{(e)}}
\end{aligned}
$$

and

$$
W_{i j}^{(e)}=\frac{\partial V_{i j}^{(e)}}{\partial z_{l}^{(e)}}
$$

Note that the partial derivatives of $\left(b_{m} b_{n}\right),\left(c_{m} c_{n}\right)$, and $\left(d_{m} d_{n}\right)$ with respect to the element vertex positions, can be determined directly from Equations (58) through (60). They are given in Table I for reference, where the quantities $\mathscr{X}_{i j}, \mathscr{Y}_{i j}$, and $\mathscr{Z}_{i j}$ are defined by

$$
\begin{aligned}
& \mathscr{X}_{i j}=x_{i}-x_{j} \\
& \mathscr{Y}_{i j}=y_{i}-y_{j} \\
& \mathscr{Z}_{i j}=z_{i}-z_{j}
\end{aligned}
$$

(Note: explicit forms of $\partial\left(b_{m} b_{n}\right) / \partial z_{l}^{(e)}$ may be obtained from Table I by replacing $\mathscr{Z}_{i j}$ with $\mathscr{Y}_{j i}$; explicit forms of $\partial\left(c_{m} c_{n}\right) / \partial x_{l}^{(e)}$ may be obtained from Table I by replacing $b_{i}$ with $c_{i}$, and $\mathscr{Z}_{i j}$ with $\mathscr{Z}_{j i}$; explicit forms of $\partial\left(c_{m} c_{n}\right) / \partial z_{l}^{(e)}$ may be obtained from Table I by replacing $b_{i}$ with $c_{i}$, and $\mathscr{Z}_{i j}$ with $\mathscr{X}_{i j}$; explicit forms of $\partial\left(d_{m} d_{n}\right) / \partial x_{l}^{(e)}$ may be obtained from Table I by replacing $b_{i}$ with $d_{i}$, and $\mathscr{Z}_{i j}$ with $\mathscr{Y}_{i j}$; explicit forms of $\partial\left(d_{m} d_{n}\right) / \partial y_{l}^{(e)}$ may be obtained from Table I by replacing $b_{i}$ with $d_{i}$, and $\mathscr{Z}_{i j}$ with $\mathscr{X}_{j i}$.)

Finally, the combined set of optimization equations, defined for the field solution unknowns in Equations (68), and the geometric discretization parameters in Equations (77)-(79), for all $e$, may be solved simultaneously for the optimal values of the field solution and geometric 
Table II. Explicit 2-D forms of $\partial\left(b_{m} b_{n}\right) / \partial y_{l}^{(e)}$ in terms of $b_{i}$ for $m, n, l=1,2,3$.

\begin{tabular}{lccc}
\hline$(m, n) / l$ & 1 & 2 & 3 \\
\hline$(1,1)$ & 0 & $2 b_{1}$ & $-2 b_{1}$ \\
$(1,2),(2,1)$ & $-b_{1}$ & $b_{2}$ & $b_{1}-b_{2}$ \\
$(2,2)$ & $-2 b_{2}$ & 0 & $2 b_{2}$ \\
$(1,3),(3,1)$ & $b_{1}$ & $b_{3}-b_{1}$ & $-b_{3}$ \\
$(2,3),(3,2)$ & $b_{2}-b_{3}$ & $-b_{2}$ & $b_{3}$ \\
$(3,3)$ & $2 b_{3}$ & $-2 b_{3}$ & 0 \\
\hline
\end{tabular}

discretization parameters. As in the 1-D case, the formulation presented above is valid for any choice of legitimate finite element basis functions.

For electromagnetic systems that possess translational symmetry, the corresponding set of 2-D optimization equations may be obtained directly from Equations (68), (77) and (78), modified to range over $l=1,2,3$, together with the following additional simplifications. First, the terms $6 \mathscr{V}_{e}$ and $36 \mathscr{V}_{e}^{2}$ appearing in the various equations and expressions in this section, should be substituted by $2 \mathscr{A}_{e}$ and $4 \mathscr{A}_{e}^{2}$, respectively, where $\mathscr{A}_{e}$ represents the area of a triangular element $e$. Second, the product term $\left(d_{m} d_{n}\right)$ as well as its partial derivatives with respect to element vertex positions, which appear in Equations (65), (72) and (75), are not required in the analogous 2-D equations. Third, it may be noted that the partial derivatives of $\left(b_{m} b_{n}\right)$ and $\left(c_{m} c_{n}\right)$ with respect to the element vertex positions, are given in Table II for 2-D systems, where $b_{i}$ and $c_{i}$ are defined as

$$
b_{i}=y_{i+1}-y_{i-1}, \quad c_{i}=x_{i-1}-x_{i+1}
$$

with the subscripts progressing modulo 3 , i.e. cyclically around the three vertices of a triangular element [22]. (Note: explicit forms of $\partial\left(c_{m} c_{n}\right) / \partial x_{l}^{(e)}$ may be obtained from Table II by replacing $b_{i}$ with $-c_{i}$.) Finally, it should be noted that the integrands in Equations (61), (62) and (63) must be reduced to their 2-D equivalents, as explained in Reference [44].

\subsection{Optimal discretization-based refinement criteria}

The most straightforward approach to developing AFEA refinement criteria based on the variational methods derived above arise directly from the optimization equations defined for the geometric discretization parameters themselves, i.e. Equation (52) for 1-D and Equations (77)-(79) for 3-D. Specifically, the relative discretization errors over a non-optimal finite element mesh can be estimated directly in terms of how well these equations are satisfied by that discretization. These optimization equations are defined element-wise, with respect to vertex positions, and they can be evaluated locally to indicate the relative 'optimality' of the elements in a mesh. All the specific optimal discretization-based refinement criteria proposed in this research are special cases derived from this fundamental template definition. As such, and unlike typical AFEA error indicators, they cannot be conveniently expressed as straightforward formulas. Rather, they are better defined and understood implicitly, as measures of the residuals of the geometric optimization equations, evaluated locally with variational field solutions on deficient finite element discretizations. It should be noted that this implicit formulation does 
not impose any significant difficulties or complexities on the implementation and evaluation of the criteria. The component terms of the optimization equations are common to standard FEA local matrix assembly modules, and thus, are readily available to facilitate the practical development and application of the new refinement criteria within electromagnetic AFEA. Two straightforward and effective examples of practical refinement estimators belonging to the family described by the template above are defined and investigated in Section 4.

\subsection{2-D second-order functional derivative criteria}

A family of functional gradient optimal discretization-based refinement criteria have been proposed for AFEA in Section 2.5, and the computational performance of specific indicators will be presented in Section 4. Despite their strengths, these first-order functional derivativebased indicators are not completely immune to the problems associated with guiding adaptive methods reliably and efficiently when used with insufficient or unbalanced discretizations $[48,49]$. Under such conditions, ineffective discretizations may evolve during the course of adaption, and poor results can occur over part, or throughout the entire adaptive process, if problematic error distributions due to unstable first-order functional derivative error indicators are not detected and corrected [44]. The purpose of this section is to investigate the use of second-order functional derivatives for analysing the stability and estimating the reliability of first-order derivative-based local error assessments.

Second-order functional derivative-based error indicators are defined in terms of derivatives with respect to element vertex positions for 2-D systems in this section. For example, in Cartesian problems where the field solution variation is independent of the co-ordinate variable $z$, i.e. $u=u(x, y)$, the second-order functional derivatives may be computed directly from the finite element optimization equations derived in Section 2.4.2. Consider a scalar triangular element with vertex positions $\left(x_{l}, y_{l}\right), l=1,2,3$. For Helmholtz systems the $x$ - and $y$-components of the second-order functional derivatives may be readily determined from the matrix forms

and

$$
\frac{1}{2} \mathbf{u}^{\mathrm{T}} \mathbf{P u}
$$

$$
\frac{1}{2} \mathbf{u}^{\mathrm{T}} \mathbf{Q u}
$$

respectively, evaluated over the elements that share the vertex in question; $\mathbf{u}$ is the field solution vector. The square matrices $\mathbf{P}$ and $\mathbf{Q}$ contain the $x$ and $y$ second-order derivative information, respectively, that corresponds to the Laplacian part of the functional for vertex $l(l=1,2,3)$ of the triangular element. The entries of the matrices $\mathbf{P}$ and $\mathbf{Q}$ are defined by

$$
P_{i j}=\frac{1}{4 \mathscr{A}_{e}^{2}} \sum_{m=1}^{3} \sum_{n=1}^{3}\left[c_{l} \frac{\partial\left(c_{m} c_{n}\right)}{\partial x_{l}}-b_{l} \frac{\partial\left(b_{m} b_{n}\right)}{\partial y_{l}}+2 \mathscr{A}_{e} \frac{\partial^{2}\left(c_{m} c_{n}\right)}{\partial x_{l}^{2}}\right] I_{i j m n}-\frac{1}{\mathscr{A}_{e}}\left(b_{l}+c_{l}\right) S_{i j}
$$

and

$$
Q_{i j}=\frac{1}{4 \mathscr{A}_{e}^{2}} \sum_{m=1}^{3} \sum_{n=1}^{3}\left[b_{l} \frac{\partial\left(b_{m} b_{n}\right)}{\partial y_{l}}-c_{l} \frac{\partial\left(c_{m} c_{n}\right)}{\partial x_{l}}+2 \mathscr{A}_{e} \frac{\partial^{2}\left(b_{m} b_{n}\right)}{\partial y_{l}^{2}}\right] I_{i j m n}-\frac{1}{\mathscr{A}_{e}}\left(b_{l}+c_{l}\right) T_{i j}
$$


where $\mathscr{A}_{e}$ is the element area; and $b_{i}$ and $c_{i}$ are the geometric parameters related to an element's vertex positions defined in Section 2.4.2. $I_{i j m n}$ is the elemental integral (in homogeneous co-ordinates) of the product of the derivatives of the $i$ th and $j$ th basis functions, with respect to the $m$ th and $n$th simplex co-ordinates, defined in Section 2.4. Note that the 'mixed' second-order functional derivative terms with respect to both the $x$ and $y$ element vertex positions are incorporated into the definitions of $\mathbf{P}$ and $\mathbf{Q}$, and that $S_{i j}$ and $T_{i j}$ are the $i j$-entries of the first-order functional derivative matrices, with respect to element vertex positions, defined in Section 2.4.2. Further, the second-order partial derivatives of $\left(b_{m} b_{n}\right)$ and $\left(c_{m} c_{n}\right)$ appearing in Equations (87) and (88) may be readily determined from Equation (84) and Table II: they evaluate to integer constants $(1,-1$ and 0$)$.

Given the first-order functional derivative quantities, these second-order results are inexpensive to compute since the only extra terms required are numerical constants which can be tabulated once and for all. As in the first-derivative case, these second-order functional derivative formulas are also valid for any choice of legitimate finite element basis functions. Further, these functional derivatives may be computed for uniform- or mixed-order meshes as may be required by specific refinement models such as in $h-, p-$, or $h p$-adaptive methods. Although these second-order results have been derived for scalar Helmholtz systems, it is interesting to note that the second-order derivatives of the wave and source terms of the functional are identically zero. This suggests that 2-D Laplace systems may benefit most from error estimation based on using both first- and second-order functional derivatives.

Second-order functional derivatives can be implemented and used as practical adaptive refinement criteria in a number of ways. For example, they can be applied in an analogous fashion to the first-order derivatives: to assess how well a discretization locally zeros these derivative quantities. In this case, the second-order derivative results can be used to verify the veracity of the first-order derivative error assessments, i.e. small first-order derivatives indicate low error (or relatively high accuracy), but large second-order derivatives suggest instability and potentially unreliable first-order derivative error results. A second approach would be to combine the two functional derivatives to form an integrated indicator, based on the first two terms of a standard Taylor's expansion. A practical electromagnetic AFEA application is presented in Section 4.2.2 to illustrate the potential advantages of this method.

\section{VERIFICATION OF OPTIMAL DISCRETIZATION FORMULATION}

As summarized in Section 1.2, a range of different formulations for determining optimal finite element discretizations have been proposed over the past 25 years. For the most part, they have been developed based on theoretical, heuristic or combined intuitive foundations. Subsequently, nearly all of these results have been shown to be incorrect or inconsistent for general applications, and of questionable veracity at best [44]. The purpose of this section is to test the validity and generality of the new formulations derived in Section 2. Independent computational investigations involving local perturbation studies and global solution space searches are reported for a range of 1-D, 2-D and 3-D electromagnetic benchmark analyses. These optimal discretization benchmarks will also be used in Section 4, to help evaluate the effectiveness and efficiency of the new refinement criteria proposed in Section 2.5. 


\subsection{1-D benchmark analyses}

A 1-D electromagnetic benchmark based on the classical free-space point charge model is presented in this section. Specifically, this point singularity model is used to determine a series of finite element approximations that possess both optimal field solutions and optimal geometric discretizations, by solving the 1-D optimization equations derived in Section 2.4. In each analysis, the objective is to resolve the functional value (stored energy) over a finite spherical annulus centred on the point charge, that spans a 100 -fold decay in electric scalar potential: the charge, of magnitude $10^{-9} / 9 \mathrm{C}$, is located at the origin; the boundaries of the problem domain are set at radial distances of 0.1 and $10 \mathrm{~m}$ from the charge. The main attribute of this system is the rapid field solution variation near the singularity. This feature is basic to many practical devices that contain sharp material corners $[22,50]$, and has been shown to drastically reduce the convergence rate of the finite element method if appropriate measures are not taken $[18,22]$. One such measure is to develop discretizations which have strongly focused distributions of DOF close to singularities, which is most readily achieved by applying AFEMs that recognize and refine regions of rapid field solution variation [20, 51].

Lagrangian basis functions were employed to approximate the unknown fields over the elements for all the 1-D solutions reported [5]. First-, second-, fourth- and eighth-order $C_{0}$ finite element approximations were tested. The optimization equations were solved using a Gauss-Newton method [52], with double-precision arithmetic throughout; and a termination convergence criterion of $10^{-10}$ was applied for both the unknown equation variables and the residuals of the optimization equations. Finally, the target functional values were calculated from the computed scalar potential solutions using exact differentiation and integration.

A series of 20 optimal first-order finite element solutions were computed. The optimal values of the discretization parameters $x_{i}$, and the field solution parameters $U_{i}$, for meshes ranging from 1 to 8 elements, are reported in Table III; the corresponding results for the other 12 meshes are tabulated in Reference [44]. Each first-order element has two discretization parameters, which define the element's vertex positions, and two field parameters, which correspond to the electric scalar potential values at these vertices. The boundary conditions were enforced by fixing the positions of the first and last vertices at 0.1 and $10 \mathrm{~m}$, and by prescribing the field solution to the analytical values of the scalar potential at those locations.

The convergence of the percent error in the functional values for these 20 discretizations is plotted in Figure 4. The corresponding errors for a series of 20 uniform first-order meshes are superposed for comparison. For all cases, the errors were calculated using the analytical functional value of $9.9000(\mathrm{~J} \mathrm{~m} / \mathrm{F})$. The optimality of these results was independently tested and fully verified by comprehensive local vertex perturbation studies and methodical global solution space searches, in order to validate the 1-D formulation derived in Section 2.4 [44].

For this benchmark system, the superior accuracy of the optimal discretization solutions relative to the uniform results is directly related to the distribution of DOF over the problem domain: the optimal formulation focuses DOF close to the point charge where the solution variation is most rapid; whereas the DOF are equally distributed throughout the space in the uniform case. For example, the relative placement of the element vertices for corresponding optimal and uniform meshes, for two, three and four elements, are described in Figure 5; the remaining 17 optimal discretizations exhibit analogous characteristics, as reported in Reference [44]. 


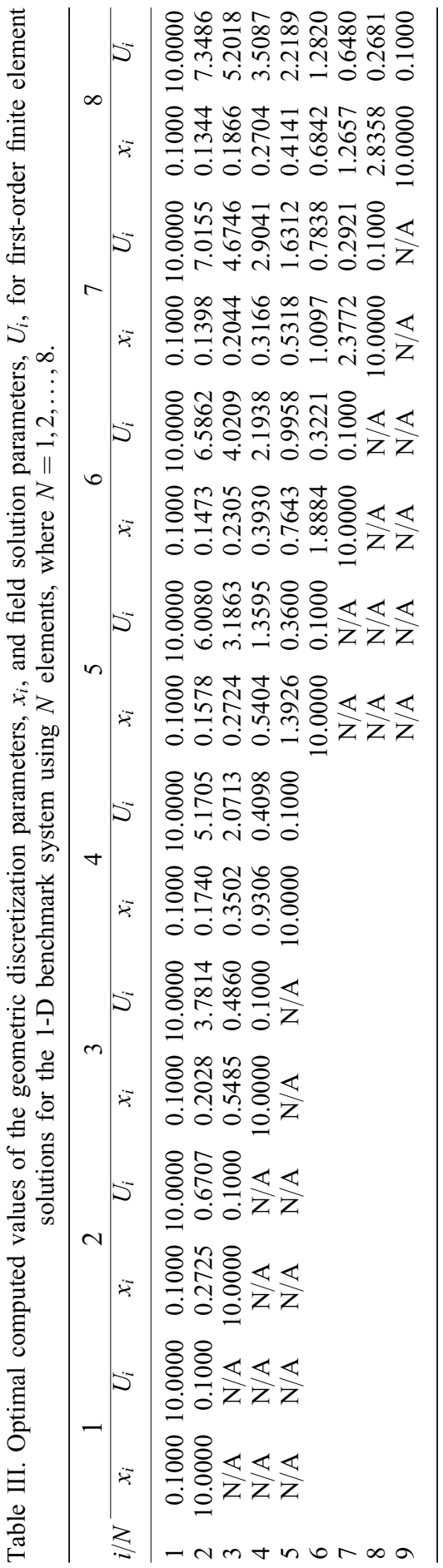




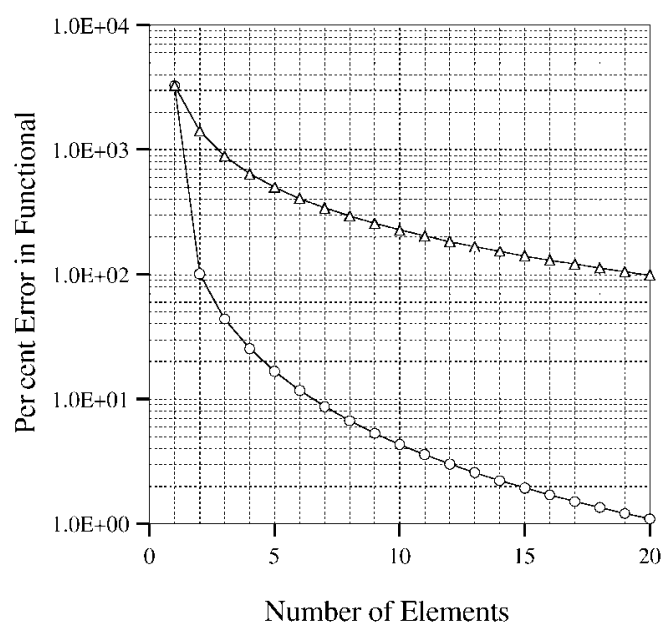

Figure 4. The variation of per cent error in functional value with discretization level for first-order finite element solutions for the 1-D electrostatic benchmark system is illustrated. The triangle knot results correspond to uniform discretizations; the circle knot results correspond to optimal discretizations.

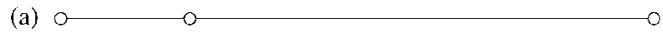

(b)
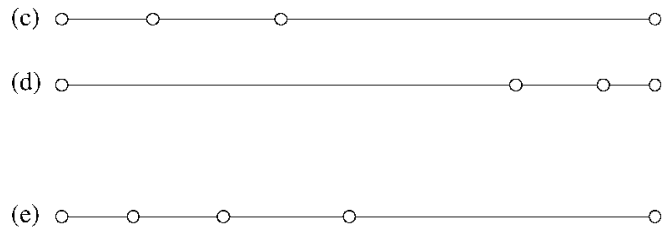

(f)

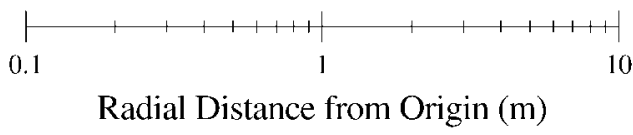

Figure 5. Example first-order optimal and uniform radial discretizations for the 1-D electrostatic benchmark system are illustrated: (a), (c), and (e) correspond to the 2, 3, and 4 element optimal meshes, respectively; (b), (d), and (f) correspond to the 2,3 , and 4 element uniform meshes, respectively. The radial discretizations are plotted on a logarithmic scale because of the proximity of the element vertices to each other near the singularity in the optimal meshes. Note: the positions of the element vertices in the optimal meshes are specified in Table III.

In addition to these first-order investigations, further series of 16, 8 and 4 optimal finite element solutions were also computed for the point charge benchmark system, using second-, fourth- and eighth-order Lagrangian elements, respectively. In each case, virtually identical conclusions were obtained [44]; therefore, only the first-order results are presented. Further, a analogous set of optimal discretization studies were conducted using a second benchmark system, based on the 1-D magnetostatic analysis of the fundamental line-singularity model. The primary results from the magnetostatic investigations are in close agreement with those reported for the electrostatic analyses; they are also reported in Reference [44]. Finally, a series of 1-D Helmholtz benchmark systems were tested, adhering to the same comprehensive procedures and independent verifications used for the singularity investigations, to validate the optimal discretization formulation for solving fundamental wave problems. These studies served to further confirm the validity of the 1-D optimization equations, and demonstrate the generality of the new optimal discretization formulation; these Helmholtz studies are reported in Reference [44].

\subsection{2-D and 3-D benchmark analyses}

A set of thirteen multidimensional singularity-based Laplacian benchmarks were solved for truly optimal discretizations in 2-D and 3-D, based on triangles and tetrahedra, using the 


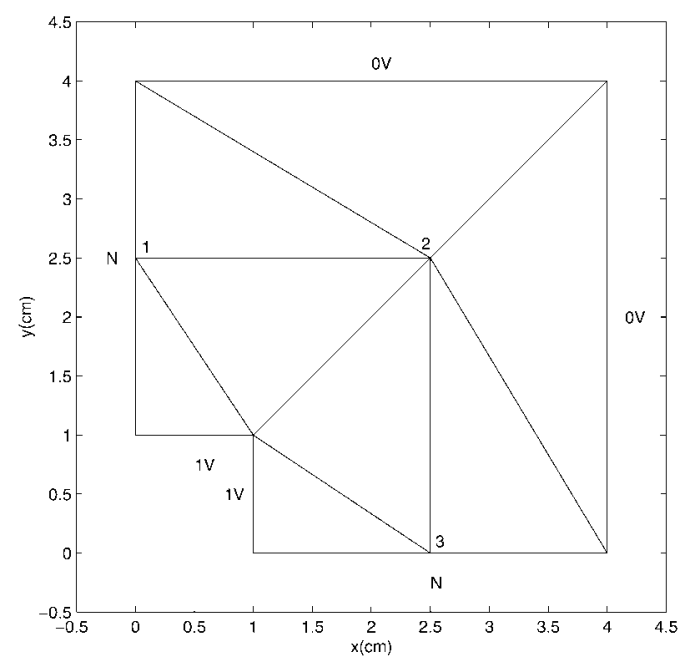

Figure 6. The geometry and initial finite element mesh configuration for the 2-D electrostatic potential analysis of the Laplace benchmark system are illustrated. Eight triangular elements are used to model one-quarter of the square coaxial line in cross-section. The conductor boundary conditions (Dirichlet) are labelled $1 \mathrm{~V}$ and $0 \mathrm{~V}$; the symmetry planes are labelled $N$ (Neumann). The sharp reentrant corner at

the intersection of the edges of the inner conductor boundary has an included angle of $270^{\circ}$.

formulations derived in Section 2.4 for 2-D and 3-D systems. For example, four of the 2-D benchmark results represent optimal $r$-refinements for the classical ' $L$ ' problem defined by Figure 6, using first-, second- , fourth- and eighth-order Lagrangian triangles, respectively. These multidimensional optimal discretization results, together with the perturbation studies and solution space searches which confirm their validity, are reported in Reference [44]. In each case, these benchmark results echo those of the 1-D investigations: they consistently affirm the validity of the new optimal discretization formulation and further demonstrate its generality. Therefore, the details of these studies have not been included here.

\section{VERIFICATION OF OPTIMAL DISCRETIZATION REFINEMENT CRITERIA}

The main purpose of this section is to evaluate the ability of the new refinement criteria, as defined in Section 2.5, to effectively and efficiently reproduce the dominant performance results associated with optimal discretizations, in actual AFEA applications. A selected set of illustrative AFEA studies, based on two practical implementations of the new criteria, are presented for applications in 1-D, 2-D and 3-D. These abridged results have been chosen to provide an unbiased representation of the full body of studies used to verify the performance of the new criteria, reported in Reference [44].

\subsection{1-D studies}

This section is presented in three parts, addressing $h$-adaption, $p$-adaption and $h p$-adaption. For each case, the optimal point-charge singularity benchmark defined in Section 3.1 is used 


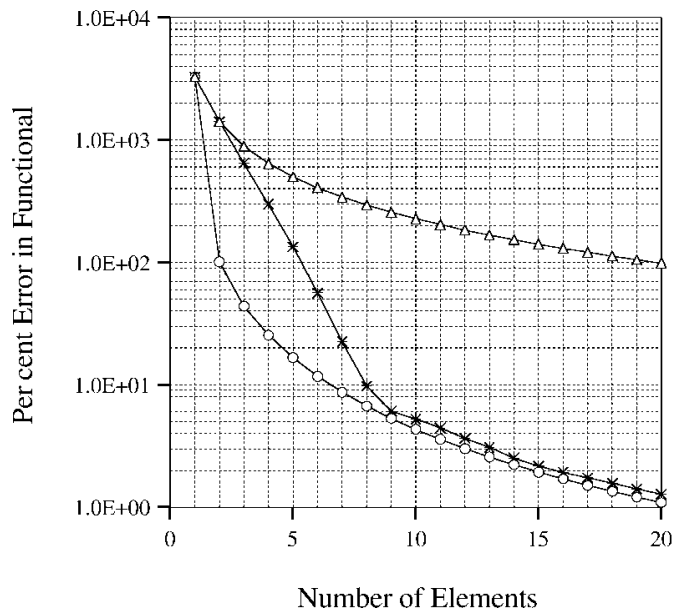

Figure 7. The convergence of per cent error in functional value with discretization level for first-order $h$-adaption studies for the 1-D electrostatic benchmark system is illustrated. The triangle knot results correspond to uniform $h$-refinement discretizations; the asterisk knot results correspond to element bisection $h$-adaption discretizations evolved using the new optimal discretization-based refinement criterion; the circle knot results correspond to first-order optimal discretizations.

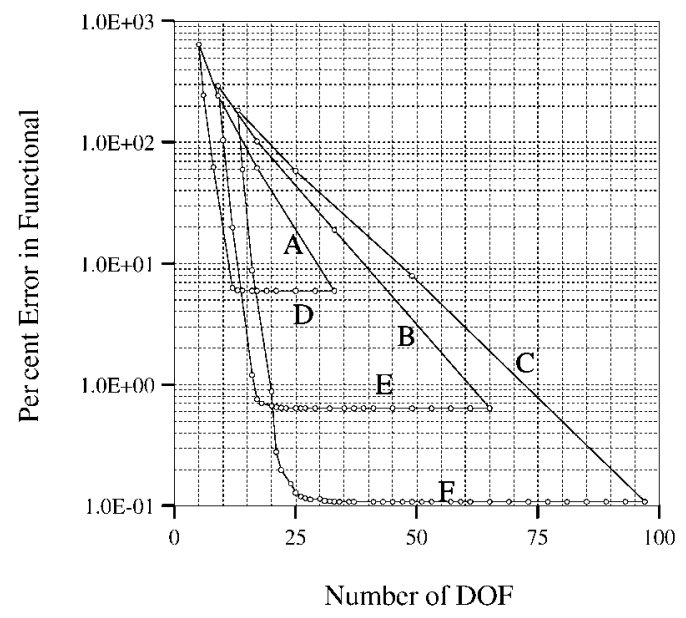

Figure 8. The convergence of per cent error in functional value with discretization level for the $p$-adaption studies for the 1-D electrostatic benchmark system is illustrated. Curve A, B, and $\mathrm{C}$ results correspond to uniform $p$-discretizations for initial meshes of four, eight and 12 elements, respectively. Curve D, E, and F results correspond to mixed-order $p$-discretizations evolved using the new optimal discretization-based refinement criterion for initial meshes of four, eight and 12 elements, respectively.

for the evaluations. All $h$-refinements were implemented using unbiased bisections; and all $p$-refinements were realized using first-, second-, fourth- and eighth-order Lagrangian elements.

4.1.1. h-adaption. The convergence of the percent error in functional value for a practical $h$-adaption strategy is illustrated in Figure 7. The initial mesh consisted of one first-order element, which was bisected to yield a uniform mesh of two elements. At each subsequent adaptive iteration, one element in the mesh was selected for bisection: the optimal discretization based refinement criterion described in Section 2.5 was used to rank the elements; the one with the highest derivative magnitude, i.e. the element which yields the largest residual for Equation (52), was chosen for refinement. The uniform $h$-refinement baseline and the optimal first-order discretization functional convergence results are also plotted in Figure 7 for comparison. The convergence of the $h$-adaption guided by the new refinement criterion indicates that it can produce discretizations that are clearly superior to uniform refinements, but also markedly inferior to initial optimal discretizations, for equivalent numbers of elements. However, after seven adaptive steps the functional accuracy achieved by the new scheme becomes remarkably close, and almost equivalent, to that of the optimal discretizations. The practical significance of this result is amplified by the relative computational cost of the two approaches: the new $h$-adaption scheme only needs to evaluate the non-linear optimization equations at fixed element vertex positions to compute the functional derivatives required for 
the adaptive refinement, which is much less expensive than solving these equations for the optimal values of the element vertex positions.

Analogous second-, fourth- and eighth-order $h$-adaption results were also computed, following the same procedures used for these first-order tests. In each case, identical conclusions were obtained with the higher-order elements [44]; therefore, only the first-order results are presented in this contribution.

4.1.2. p-adaption. The convergence of the percent error in functional value for two $p$-adaption strategies, applied to a range of uniform initial meshes, is illustrated in Figure 8. Uniform and mixed-order $p$-adaption schemes were investigated. The performance of uniform $p$-adaption for initial meshes of four, eight and 12 elements is shown by curves $\mathrm{A}, \mathrm{B}$ and $\mathrm{C}$, respectively. In each case, the initial discretizations were comprised of firstorder elements, which were successively refined by incrementing all the element orders uniformly, through to eighth order. The functional convergence for the corresponding mixed-order $p$-adaption scheme is represented by curves $\mathrm{D}, \mathrm{E}$ and $\mathrm{F}$, for the four, eight and 12 element meshes, respectively. For the mixed-order $p$-adaption, only one element in the mesh was refined at each adaptive iteration; the derivative magnitude criterion described Section 4.1.1 was used to select the best candidate. If an eighth-order element was chosen for refinement, the next highest ranking lower-order element was refined instead. Based on Figure 8, mixed-order $p$-adaption results in a significantly faster rate of convergence, compared to uniform $p$-adaption, for uniform initial meshes. For example, in the four element case, the same functional accuracy level was achieved with $13 \mathrm{DOF}$ by mixed-order $p$-adaption, compared to $33 \mathrm{DOF}$ for the uniform method (a relative savings of approximately 61 per cent in the number of DOF). Similarly, for the eight element case a relative reduction of approximately 66 percent in the number of DOF was achieved using mixed-order $p$-adaption, compared to uniform $p$-refinements. Analogously, a 68 percent economy in DOF was realized in the 12 element case.

4.1.3. hp-adaption. The convergence of the percent error in functional value for three primary $h p$-adaption strategies, based on integrated, decoupled and uniform $h p$-refinements are investigated. The objective for these studies is not only to evaluate the effectiveness of the new refinement criterion for $h p$-adaption, but also to determine which type of $h p$-adaption model is more efficient for resolving point singularities. At each step, the integrated $h p$-adaptive strategy refined the discretization by either bisecting an element or increasing its order. The decoupled approach started by refining the first-order mesh using element bisection for a fixed number of steps, and then continued by increasing the order of individual elements in each subsequent adaptive refinement (i.e. mixed-order $p$-adaption). For both adaption models, elements were ranked for refinement using the same derivative magnitude criterion employed for the individual $h$ - and $p$-adaption investigations, and the mixed-order $p$-refinements were implemented using the same procedures adopted in Section 4.1.2. The uniform $h p$-adaption results were also determined using both integrated and decoupled approaches. For the integrated approach, all of the elements in a mesh were bisected, and their orders augmented, alternately, at successive adaptive iterations. For the decoupled approach, the first-order mesh was refined by element bisection for the first three adaptive steps, then the element orders were increased uniformly at each successive step. 


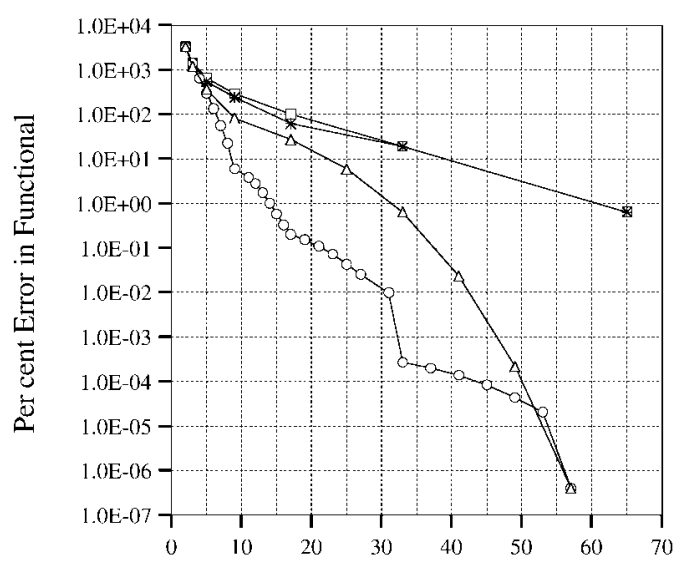

Number of DOF

Figure 9. The convergence of per cent error in functional value with discretization level for $h p$-adaption studies for the 1-D electrostatic benchmark system is illustrated. The triangle knot results correspond to integrated $h p$-discretizations evolved using the new optimal discretization-based refinement criterion; the circle knot results correspond to decoupled $h p$-discretizations evolved using the new optimal discretization-based refinement criterion; the asterisk knot results correspond to uniform integrated $h p$-refinement discretizations; the square knot results correspond to uniform decoupled $h p$-refinement discretizations.

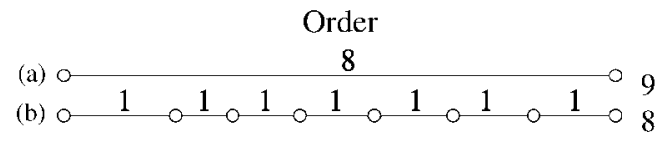

$\begin{array}{lllllllll} & 8 & 8 & \circ & 17 \\ \text { (a) } 0 & 4 & 2 & 2 & 2 & 2 & 2 & 2 & 17\end{array}$

(a) $\bigcirc \quad 8 \quad 0 \quad 8 \quad 0 \quad 8025$

(b) $\square \quad 4 \circ 4 \circ \quad 4 \circ 4 \circ \quad 4 \circ 2 \circ \quad 2 \circ 25$

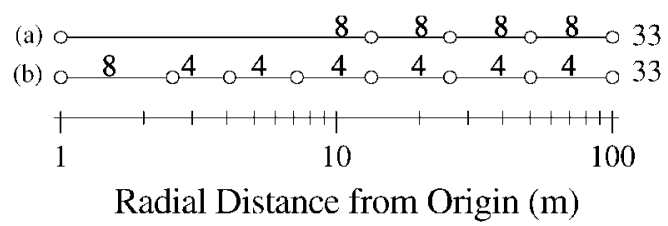

Figure 10. Evolving radial discretizations for $h p$-adaption for 1-D electrostatic benchmark system are illustrated: (a) integrated $h p$-adaption discretizations; and (b) decoupled $h p$-adaption discretizations. The radial discretizations are plotted on a logarithmic scale because of the proximity of the element vertices to each other near the singularity. Note: the positions of the element vertices in the meshes are determined by element bisection; the orders of the elements are specified above each element; and the number of DOF in each mesh is shown to the right of each discretization.

The convergence of the percent error in functional value for these $h p$-adaption strategies are illustrated in Figure 9. Note that the variable refinement integrated and decoupled schemes result in superior rates of convergence relative to the corresponding uniform $h p$ adaption strategies for this benchmark system. Further, the decoupled approach is seen to provide a faster rate of convergence relative to integrated $h p$-adaption. Theoretically, decoupled $h p$-adaption should not yield faster convergence than fully integrated $h p$-adaption [44]. However, based on the point singularity results, the decoupled approach is more effective for systems with regions of rapid field variation. The superior decoupled $h p$-adaption performance may be explained as follows. The decoupled and integrated $h p$-adaption schemes evolve significantly different discretizations, as illustrated in Figure 10. The integrated approach initially attempts to resolve the singularity by increasing element order, rather than by element bisection. The decoupled approach yields a superior distribution of DOF primarily because it produces meshes with a higher density of DOF near the singularity, compared with the less tightly focussed distribution produced by the integrated approach.

4.1.4. Discussion. The adaption examples presented in the sections above have been chosen to represent a range of the basic methods most commonly used for practical electromagnetic 
AFEA. However, to confirm the highest possible resolution in the convergence rates, each adaptive iteration was implemented to increase the number of DOF in a discretization by the minimal increments appropriate for the adaption model examined. In addition, analogous evaluations were computed for two other benchmark systems: the magnetostatic line-current singularity model; and the 1-D Helmholtz transmission-line model. The results of these investigations are consistent, and strongly supportive of those obtained in the electrostatic studies; they are presented in Reference [44]. Therefore, based on these $h$ - , $p$ - and $h p$-adaption performance evaluations, it is clearly indicated that the newly proposed adaptive refinement criteria have the potential to effectively and efficiently reproduce the dominant performance characteristics associated with optimal finite element discretizations, in practical electromagnetic AFEA applications. Performance evaluations for similar optimal discretization-based refinement criteria, for the AFEA of 2-D and 3-D electromagnetic systems are reported in Sections 4.2 and 4.3.

\subsection{2-D studies}

A series of studies based on a fundamental Laplace benchmark system is investigated to evaluate the computational performance of the new AFEA refinement criteria within $h^{-}$, $p$ - and $h p$-adaptive solvers. Two practical implementations of these criteria are tested and compared against some of the most effective and efficient indicators available. Unlike the 1-D studies, the hierarchal basis functions developed in Reference [53] are used to approximate the unknown field solutions, over the $C_{0}$ triangular elements, for the tests reported in this section.

The most direct equivalent to the 1-D functional derivatives applied in Section 4.1 are the gradients of the functional, with respect to the element vertex positions, in a 2-D mesh. Therefore, a simple extension of the 1-D criterion is to use a weighted sum or an average value of the magnitudes of the vertex-based functional gradients associated with a triangle; this implementation is called Type-A criterion. A more directed approach is to use the projections of the functional gradients onto vectors directed from the vertices towards the centroids of the triangles; this version is called Type-B criterion. Unlike Type-A approach, this implementation incorporates both the directions and the magnitudes of the functional gradients.

4.2.1. Laplace studies. The Laplace studies are based on the standard electrostatic ' $\mathrm{L}$ ' problem defined in Figure 6. It consists of one-quarter of an infinitely long, air-filled, uniform, square coaxial line modeled in cross-section. The objective for this benchmark is to compute the functional value corresponding to the electrostatic potential energy per unit length stored in the air region between the two conductors. The dominant feature of this system is the rapid field solution variation close to the sharp reentrant corner. This feature is common to many practical devices that contain sharp material edges, and has been shown to significantly reduce the convergence rate of the finite element method [5].

The convergence of the percent error in functional value for first-order $h$-adaption is illustrated in Figure 11. The initial mesh used for these studies is defined by Figure 6. The Type-A refinement criterion was used to rank the elements at each adaptive step; and the elements with the highest weighted-average gradient magnitudes were chosen for refinement. Further, a 50 per cent increment in the number of DOF per adaptive refinement was used to update the discretizations. In addition, all $h$-refinements were based on either element 


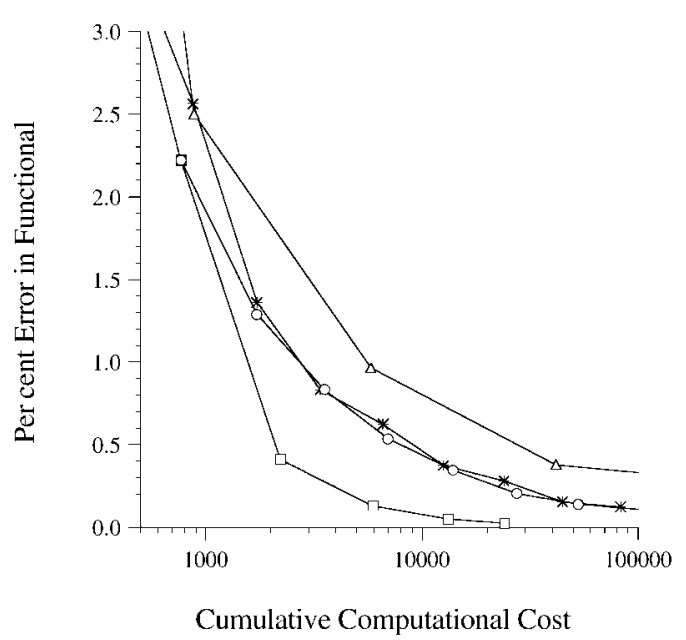

Figure 11. The convergence of per cent error in functional value with discretization level for first-order $h$ - and $h p$-adaption studies for the Laplace benchmark system is illustrated: the triangle knot results correspond to uniform discretizations; the circle knot results correspond to $h$-adaption discretizations evolved using the new optimal discretization-based refinement criteria (Type-A); the asterisk knot results correspond to $h$-adaption discretizations evolved using field discontinuity-based refinement criteria; the square-knot results correspond to $h$-followed by mixed-order $p$-adaption (i.e., $h p$-adaption) discretizations evolved using the new optimal discretization-based refinement criteria (Type-A). Note: the cumulative computational cost of adaption was calculated based on using a preconditioned conjugate gradient algorithm to solve the finite element matrix equations.

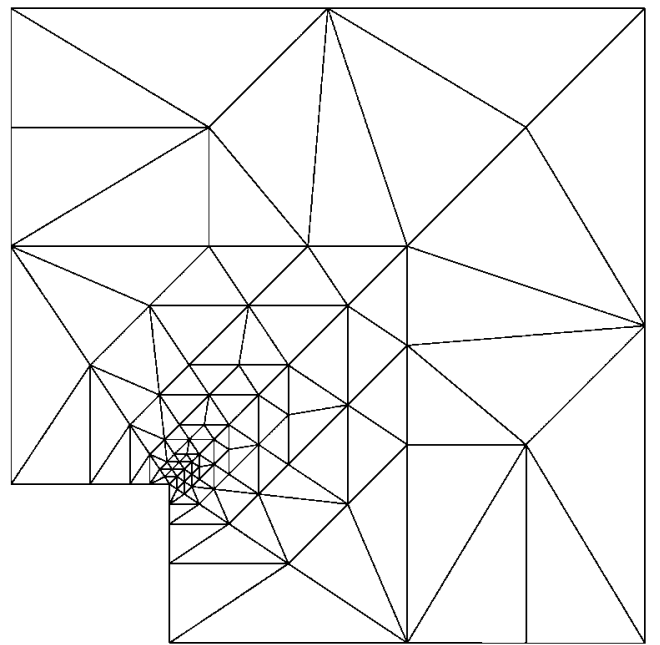

Figure 12. An example refinement due to the new $h$-adaption is illustrated for the Laplace benchmark system. The second-order discretization shown was evolved using the new optimal discretization-based refinement criteria (Type-A). The initial mesh used for the $h$-adaption is the eight-element discretization defined by Figure 6 . The $h$-refinements at each adaptive iteration were based on element bisections and uniform subdivisions of elements into similar triangles. The resulting set of new element vertices were retriangulated using a Delaunay algorithm.

bisections or 1:4 similarity subdivisions; however, the resulting set of new element vertices were retriangulated after each adaptive step using a Delaunay algorithm [54]. Both the uniform $h$-refinement baseline, and a practical field discontinuity $h$-adaption result [21] are also plotted in Figure 11 for comparison. It should be noted that this field discontinuity refinement criterion has been shown to be amongst the most effective for resolving AFEA problems of this type using $h$-adaption models [55]. Analogous second-, fourth- and eighth-order $h$-adaption results for the Laplace benchmark system are reported in Reference [44]; they were computed following the same procedures used for the first-order tests. Overall, $h$-adaption guided by the optimal discretization-based refinement criteria consistently produced results of significantly superior accuracy, compared to uniformly refined meshes with similar numbers of DOF, in each analysis. Finally, an example $h$-adapted mesh is presented in Figure 12 to illustrate the sharp focus of DOF near the reentrant corner. 
Table IV. Discretization level versus per cent error in functional for $p$-adaption strategies applied to the Laplace benchmark system.

\begin{tabular}{lcccr}
\hline Method/\#DOF & $1.00 \%$ & $0.50 \%$ & $0.10 \%$ & $0.05 \%$ \\
\hline Uniform p-adaption & 230 & 290 & 1050 & 1670 \\
Hierarchal coeff. $p$-adaption & 230 & 290 & 530 & 675 \\
New $p$-adaption & 110 & 200 & 550 & 700 \\
New $h$-adaption & 100 & 120 & 280 & 375 \\
\hline
\end{tabular}

The performance results for a range of $p$-adaption strategies applied to the Laplace benchmark system are summarized in Table IV. Specifically, uniform and mixed-order $p$-adaption schemes were investigated using $C_{0}$ hierarchal elements ranging from order one through 10 [56]. An initial mesh of 128 first-order elements, defined by uniformly subdividing the eight element mesh shown in Figure 6, was used for the $p$-adaption studies. In addition to the uniform $p$-refinement baseline, the hierarchal coefficient $p$-adaption result [51] is presented for comparison. It should be noted that the hierarchal coefficient criterion has been shown to be amongst the most effective for resolving AFEA problems of this type using $p$-adaption [55]. Type-A refinement criterion was used to rank the elements, and those in need of refinement were identified as before. The order of an element selected for refinement was increased successively from first- through to tenth-order each time the element was selected to be refined; however, if a tenth-order element was chosen for refinement, the highest ranking lower-order element was refined instead. Further, a 50 per cent increment in the number of DOF per adaptive refinement was used to update the discretizations. Based on Table IV, it is clear that for uniform-order initial meshes, the new mixed-order $p$-adaption scheme results in significantly faster rates of convergence compared to uniform $p$-adaption. The same functional accuracy was achieved with 700 DOF by the new mixed-order $p$-adaption scheme, compared against $1670 \mathrm{DOF}$ for the uniform method (a relative savings of approximately 58 per cent in the number of DOF). Further, it should be noted that the new mixed-order $p$-adaption performance results are on par with the hierarchal coefficient results. An example $p$-adapted mesh is presented in Figure 13 to illustrate the strongly focussed and efficient placement of the higher-order elements near the reentrant corner.

The performance results for combined $h p$-adaption are also reported in Table IV. The decoupled $h p$-adaptive strategy first refined the initial first-order eight-element mesh shown in Figure 6 by $h$-adaption for the first three adaptive steps, and then continued to refine the discretization using mixed-order $p$-adaption in each subsequent step. For both adaption models, elements were ranked for refinement using Type-A criterion, and the specific $h$ - and $p$-adaptive refinements were implemented using the same procedures described earlier for the individual $h$ - and $p$-adaption studies. The convergence of the percent error in functional value for the decoupled $h p$-adaption strategy is clearly superior to all of the $p$-adaption strategies investigated for the Laplace benchmark system. In addition, these $h p$-adaption results are also plotted in Figure 11, where it is readily confirmed that they are clearly superior to all the methods represented therein.

In addition to the $h$ - , $p$ - and $h p$-adaption Laplace benchmark studies presented above, comprehensive analogous investigations were conducted for a Helmholtz benchmark, based on the 2-D time-harmonic analysis of a planar microstrip antenna. Type-B refinement criterion 


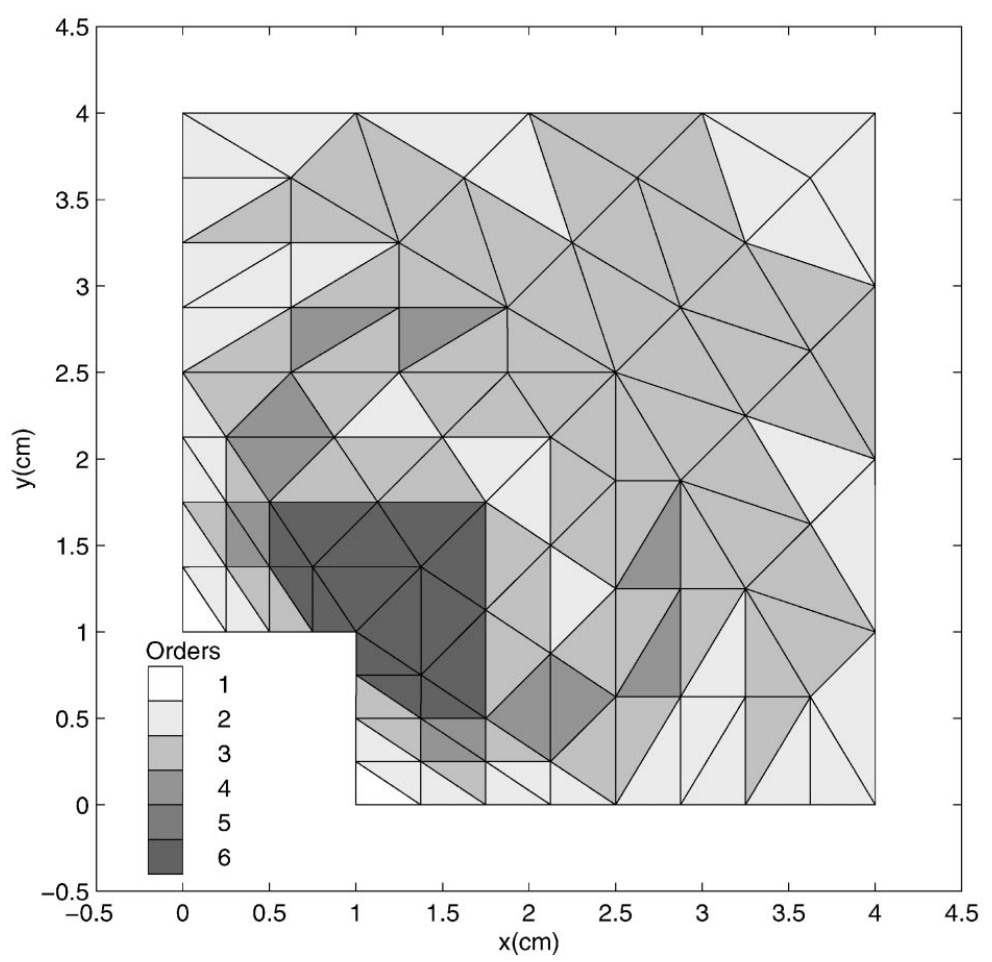

Figure 13. An example refinement due to the new $p$-adaption is illustrated for the Laplace benchmark system. The mixed-order discretization shown was evolved using the new optimal discretization-based refinement criteria (Type-A). The initial discretization used for the $p$-adaption is the 128 element first-order mesh defined by the figure. The range of element orders used in the discretization are shown in the legend accompanying the figure.

was used for these studies, and the results are consistent with, and supportive of, those found for the Laplace system; they are reported in Reference [44]. Overall, these investigations serve to further confirm the general applicability, effectiveness and efficiency of the newly proposed optimal discretization-based refinement criteria for practical AFEA applications in 2-D electromagnetics.

4.2.2. Second-order derivative criteria. The purpose of this section is to illustrate the potential value of using second-order functional derivative indicators in 2-D electromagnetic AFEA applications. The test system is defined by Figure 6. Performance results for second-order (i.e. using quadratic $C_{0}$ triangles) $h$-adaption studies on functional convergence are presented in Figure 14. The uniform $h$-refinement baseline is included for comparison with $h$-refinement based on both a first-order derivative error estimator (Type-A) and an integrated (Taylor's expansion) first- and second-order error criterion (Type-A). A 50 per cent increment in the number of DOF per adaptive step was used to update the discretizations. The results indicate a marginal improvement in performance for functional accuracy levels between 1 and 0.1 per cent, for the integrated criterion versus the first-order derivative estimator, and an increasingly 


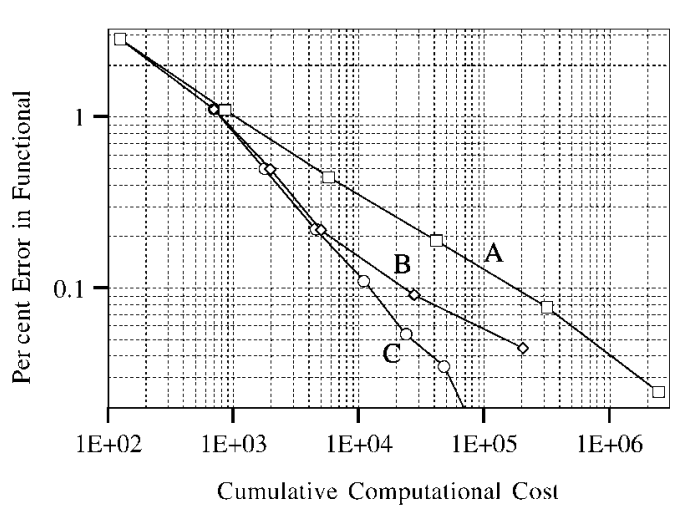

Figure 14. The convergence of per cent error in functional value with discretization level for second-order (i.e., quadratic $C_{0}$ elements) $h$-adaption studies with 50 per cent DOF updates for the 2-D Laplace benchmark system is illustrated: the curve (A) results correspond to uniform discretizations; the curve (B) results correspond to $h$-adaption discretizations evolved using a first-order derivative error estimator (Type-A); the curve (C) results correspond to $h$-adaption discretizations evolved using a combined firstand second-order error estimator (Type-A). Note: The cumulative computational cost of adaption was calculated based on using a preconditioned conjugate gradient algorithm to solve the finite element matrix equations.

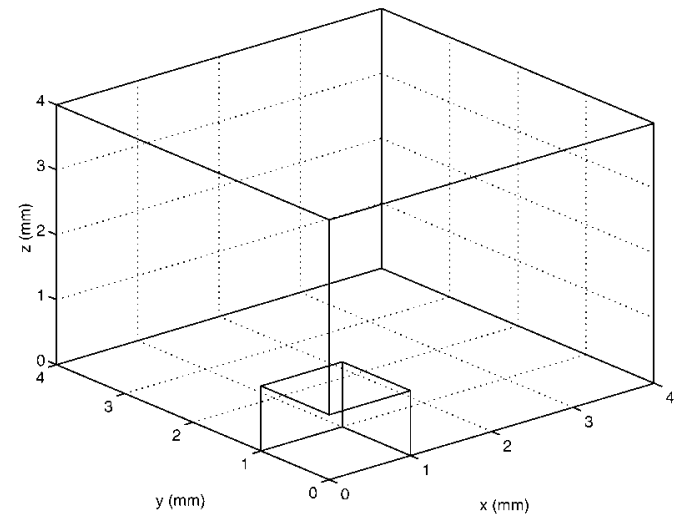

Figure 15 . The geometry used for the 3-D electrostatic potential analysis of the Laplace benchmark system is illustrated. The figure depicts one-eighth of an air-filled, concentric, cuboidal capacitor. The conductor boundary conditions are $1 V$ (Dirichlet) on the smaller, inner, ideal conductor cube and $0 \mathrm{~V}$ (Dirichlet) on the outer, ideal conductor cube. The symmetry planes defined by $x=0, y=0$, and $z=0$ were left unconstrained (Neumann) between the two ideal conductors.

significant improvement for functional accuracy levels beyond 0.1 per cent. In addition to this study, analogous tests based on the microstrip antenna benchmark addressed in Section 4.2.1 were also conducted, using Type-B criterion. Overall, these results demonstrate that integrated estimators, formulated from first- and second-order functional derivatives, have the potential to improve upon the AFEA performance of purely first-order indicators.

\subsection{3-D study}

The purpose of this section is to investigate the potential benefits and related costs of using the finite element optimization equations, (77)-(79), as optimal discretization-based refinement criteria for 3-D electromagnetic AFEA. Similar to the 2-D development, functional gradient error indicators for 3-D refinement criteria can be defined directly in terms of derivatives with respect to tetrahedral vertex positions. Analogous to the 2-D result, one straight forward technique is to assess a weighted sum of vertex-based functional gradient magnitudes for each element, then use these values to rank the elements for refinement. This method is illustrated in the following example, based on the 3-D test problem defined by Figure 15. This benchmark represents one-eighth of an air-filled, concentric, cuboidal capacitor, i.e. the 3-D analog to the classical 2-D ' $\mathrm{L}$ ' problem. The objective for this study is to compute the functional 


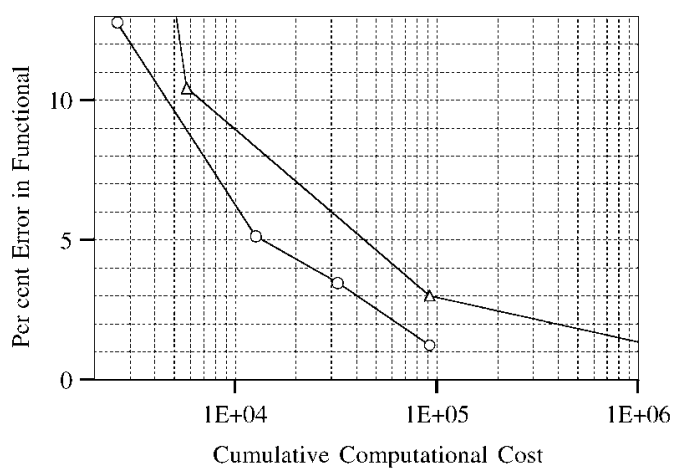

Figure 16. The convergence of per cent error in functional value with discretization level for first-order $h$-adaption studies for the Laplace benchmark system are illustrated: the triangle knot results correspond uniform $h$-refinements; the circle knot results correspond to $h$-adaption discretizations evolved using the new optimal discretization-based refinement criterion. Note: the cumulative computational cost of adaption was calculated based on using a preconditioned conjugate gradient algorithm to solve the finite element matrix equations.

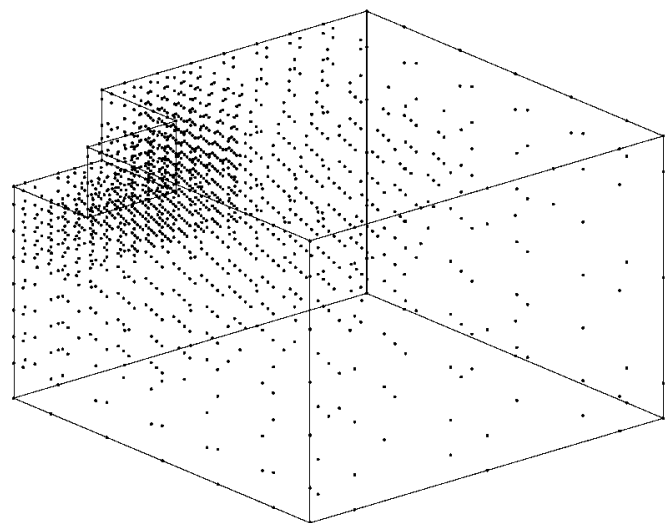

Figure 17. An example refinement achieved using the new $h$-adaption is illustrated, in terms of the distribution of tetrahedra vertices, for the Laplace benchmark system. The first-order discretization represented above was evolved using the new optimal discretization-based refinement criterion. The initial mesh used for the $h$-adaption was comprised of 144 elements. Note: the figure depicts the same one-eighth of the cuboidal capacitor considered previously, but viewed from a different perspective in order to effectively illustrate the distribution of element vertices.

value corresponding to the electrostatic potential energy stored in the air region between the conductors. The primary feature of this system is the rapid field solution variation close to the sharp reentrant edges and corners of the inner conductor. These features are common to many practical devices, and have been shown to significantly reduce the convergence rate of the finite element method [57, 58].

The convergence of the percent error in functional value for $h$-adaption is illustrated in Figure 16. The initial mesh used for these studies was comprised of 144 first-order Lagrangian tetrahedra. At each subsequent adaptive step, the new refinement criterion was used to rank the elements, and the tetrahedra with the highest average functional gradient magnitudes were chosen for refinement. A 100 per cent increment in the number of DOF per adaptive refinement was used to update the discretizations. The $h$-refinements were implemented using the tetrahedral mesh refinement algorithms described in Reference [59]. The uniform $h$-refinement baseline is also included in Figure 16 for comparison. These results show that the new $h$-adaption strategy produced significantly higher accuracy levels than uniform discretizations, at comparable computational costs. For example, for solution accuracies near 1 per cent, more than a 10 -fold savings in computational cost is achieved. Finally, an example $h$-adapted 3 -D discretization is given in Figure 17, to illustrate the sharp focus of DOF produced by the new refinement criterion near the reentrant edges and corners of the inner conducting cube. 


\subsection{Switched-reluctance motor study}

Switched reluctance motor (SRM) operation depends on how reluctance paths change with rotor position, for torque production; they are commonly found in applications requiring precision movements. The purpose of this section is to demonstrate a practical application of the new Type-A refinement criterion for the 2-D $h$-adaptive FEA of the SRM design represented by Figure 18. Although standard, non-adaptive, magnetostatic FEMs can be used to analyse this SRM, key features of the device make it challenging and computationally intensive to determine its field solution(s) accurately. For example, the design of SRMs requires the calculation of the magnetic flux linkage of the motor as a function of both rotor position and excitation current [60]. To analyse the full behaviour of this SRM, at least eight solutions are required at different angular positions of the rotor, and for each position, at least eight different excitation current levels need to be considered [60]. Therefore, a practical design cycle will involve a minimum of 64 solutions, and a new finite element discretization must be created for each rotor position. Further, the complex geometric details of the machine must be adequately resolved by the finite element discretizations to yield accurate solutions. For example, the narrow air gap regions and the curved material boundaries of the design must be modelled sufficiently well with appropriate numbers and sizes of elements. Moreover, several refinements of an initial discretization may be necessary to converge the finite element field solution errors to within practical engineering tolerances. Consequently, a large number of DOF can be required to compute each of the 64 field solutions accurately, and AFEMs are frequently used for efficient SRM analysis and design.

The convergence of the percent error in functional value for the $h$-adaptive analysis of the SRM is illustrated in Figure 19. The initial mesh was comprised of 257 first-order elements, and 50 per cent DOF increments were used to update the discretizations in each subsequent adaptive step. The uniform $h$-refinement baseline result, starting from the same initial mesh, is also plotted for comparison. A termination criterion of 2.5 per cent error in the functional value was used for both adaption runs. The new $h$-adaption provides a considerable cost reduction over the uniform approach: a functional accuracy level of approximately 2.8 per cent is achieved after four adaptive steps, at a relative computational cost of 24,452; whereas, the error in the functional value after three uniform refinements is approximately 3.4 per cent, at a relative computational cost of 109869 . These figures indicate a 78 per cent reduction in the computational effort required to converge the field solution to a functional error of 5 per cent error. Similarly, a savings of 94 per cent was appreciated for functional accuracy levels of less than 2.5 per cent error. The significance of these savings is amplified by the fact that 64 finite element field solutions are required for this SRM analysis. Finally, a plot of the SRM magnetic flux density is provided in Figure 20, to illustrate the highly non-uniform field variation over the device.

\section{CONCLUSIONS}

The central goal of this research has been to develop practical adaptive refinement criteria which can make it possible for AFEMs to effectively reproduce the dominant and essential modelling characteristics of optimal finite element discretizations, without the prohibitive computational costs and complexities associated with constructing optimal discretizations. Four 


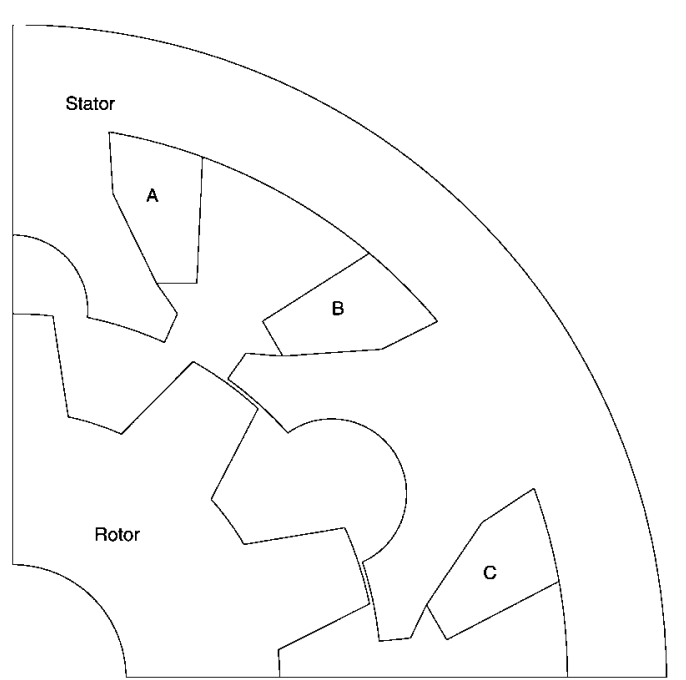

Figure 18. The geometry for the magnetic vector potential analysis of a switched reluctance motor is illustrated. The 2-D view depicts one-quarter of the cross-section of a 12/10 switched reluctance motor in an unaligned position [54]. The air gap between the stator and rotor is $0.5 \mathrm{~mm}$, and the total diameter of the motor is $165 \mathrm{~mm}$. The stator and rotor relative permeabilities are each 1000, and the coil labelled $\mathrm{A}$ was excited with a uniform current density of $1.0 \mathrm{~A} / \mathrm{m}^{2}$.

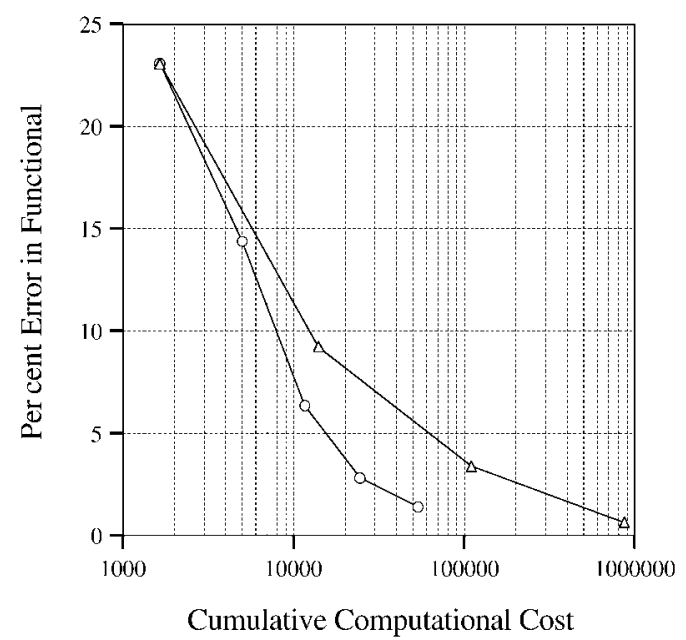

Figure 19. The convergence of per cent error in functional value with discretization level for first-order $h$-adaption studies for the switched reluctance motor model of Figure 18 is illustrated. The triangle knot results correspond to per cent error in functional values computed from solutions based on first-order uniform discretizations. The circle knot results correspond to per cent error in functional values computed from solutions based on first-order $h$-adaption discretizations evolved using the new optimal discretization-based refinement criteria (Type-A). Both results are based on the same initial mesh. Note: the cumulative computational cost of adaption was calculated based on using a preconditioned conjugate gradient algorithm to solve the finite element matrix equations.

key contributions are provided by this work: the first three are primary to the central objective; the fourth is secondary, but of equal importance to the research area in general. First, a new formulation for building truly optimal finite element discretizations for electromagnetics analysis has been developed. It was derived from a variational foundation; cross-verified and authenticated on a theoretical level; and independently validated using rigorous computational testing. The result is valid for scalar Laplace, Poisson, Helmholtz and eddy-current diffusion problems, modeled in 1-D, 2-D and 3-D. Second, a template prescription for implementing a family of optimal discretization-based AFEA refinement criteria has been developed. Two practical implementations were derived, evaluated and shown to be highly effective, cost-efficient and potentially superior to the state-of-the-art. Third, both second-order and mixed-order functional derivative error indicators, designed to identify, stabilize and improve spurious and unreliable adaptive refinement trajectories, have been developed. An example mixed-order implementation was presented, tested and shown to be very effective in a 


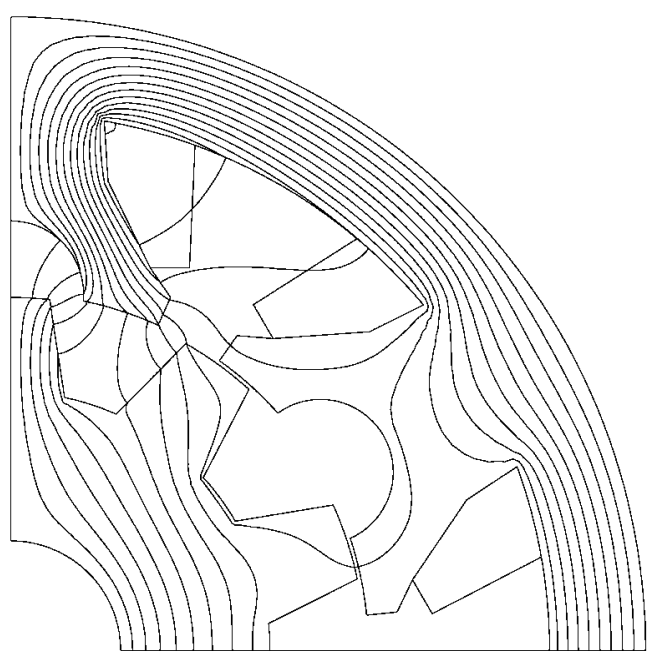

Figure 20. An approximate field solution for the switched reluctance motor model of Figure 18 is illustrated. The plot is based on the finite element solution computed using an adaptively refined mesh with 1847 second-order elements.

sensitive electromagnetic AFEA application. Finally, a set of fundamental electromagnetic FEA benchmark systems with truly optimal finite element discretizations has been developed and fully validated. This primary test bed provides an objective and absolute measure by which to assess the raw performance and effectiveness of any adaptive refinement criterion considered for electromagnetics AFEA applications. Overall, the consistent and strongly supported conclusion of this research study is that the new optimal discretization-based refinement criteria possess the potential to facilitate the development of practical AFEMs that can reproduce the dominant and essential modelling aspects of optimal discretizations, without incurring their associated costs or complexities.

\section{REFERENCES}

1. Silvester PP. Finite element solution of homogeneous waveguide problems. Alta Frequenza 1969; 38:313-317.

2. Silvester PP. High-order polynomial triangular finite elements for potential problems. International Journal of Engineering Science 1969; 7:849-861.

3. Silvester PP. A general high-order finite-element waveguide analysis program. IEEE Transactions on Microwave Theory and Techniques 1969; 17:204-210.

4. Ahmed S, Daly P. Waveguide solutions by the finite element method. Radio and Electronic Engineer 1969; 38:217-223.

5. Silvester PP, Ferrari RL. Finite Elements for Electrical Engineers (2nd edn). Cambridge University Press: Cambridge, 1990.

6. Ida N, Bastos JPA. Electromagnetics and Calculation of Fields. Springer: New York, 1992.

7. Jin J. The Finite Element Method in Electromagnetics. Wiley: New York, 1993.

8. Calamia M, Pelosi G, Silvester PP (eds). Second International Workshop on Finite Element Methods for Electromagnetic Wave Problems: Siena, Italy, May 1994. James and James: London, UK, 1994.

9. Webb JP. Application of the finite-element method to electromagnetic and electrical topics. Reports on Progress in Physics 1995; 58:1673-1712.

10. Lowther DA, Silvester PP. Computer-Aided Design in Magnetics. Springer: New York, 1986.

11. Sabonnadiere JC, Coulomb JL. Finite Element Methods in CAD: Electric and Magnetic Fields. Springer: New York, 1987. 
12. Hoole SRH. Computer-Aided Analysis and Design of Electromagnetic Devices. Elsevier Science Publishing Company: New York, 1989.

13. Gandhi OP (ed.). Biological Effects and Medical Applications of Electromagnetic Energy. Prentice-Hall: Englewood Cliffs, NJ, 1990.

14. Wickelgren I. The strange senses of other species. IEEE Spectrum 1966; 33(3):32-37.

15. Cendes ZJ. Em simulators: Cae tools. IEEE Spectrum 1990; 27(11):73-77.

16. Sabonnadiere JC, Konrad A. Computing em fields. IEEE Spectrum 1992; 29(11):52-56.

17. Mackerle J. Error analysis, adaptive techniques and finite and boundary elements - A bibliography (1991-1993). Finite Elements in Analysis and Design 1994; 17:231-246.

18. Zienkiewicz OC, Taylor RL. The Finite Element Method, vol. 1: Basic Formulation and Linear Problems (4th edn). McGraw-Hill Book Company: London, 1989.

19. McFee S, Giannacopoulos D. Optimal discretization based refinement criteria for finite element adaption. IEEE Transactions on Magnetics 1996; 32(3):1357-1360.

20. Giannacopoulos D, McFee S. Towards optimal $h-p$ adaption near singularities in finite element electromagnetics. IEEE Transactions on Magnetics 1994; 30(5):3523-3526.

21. Webb JP, Forghani B. Adaptive improvement of magnetic fields using hierarchal tetrahedral finite elements. IEEE Transactions on Magnetics 1994; 30(5):3511-3514.

22. Silvester PP, Ferrari RL. Finite Elements for Electrical Engineers (3rd edn). Cambridge University Press: Cambridge, 1996.

23. Denny VE, Landis RB. A new method for solving two-point boundary value problems using optimal node distribution. Journal of Computational Physics 1972; 9:120-137.

24. Carroll WE, Barker RM. A theorem for optimum finite-element idealizations. International Journal of Solids and Structures 1973; 9:883-895.

25. Prager W. A note on the optimal choice of finite element grids. Computer Methods in Applied Mechanics and Engineering 1975; 6:363-366.

26. Tang JW, Turcke DJ. Characteristics of optimal grids. Computer Methods in Applied Mechanics and Engineering 1977; 11:31-37.

27. Masur EF. Some remarks on the optimal choice of finite element grids. Computer Methods in Applied Mechanics and Engineering 1978; 14:237-248.

28. Babuska I, Rheinboldt WC. Analysis of optimal finite element meshes in $R_{1}$. Mathematics of Computation 1979; 33(146):435-463.

29. Miller K, Miller RN. Moving finite elements: Parts 1 and 2. SIAM Journal on Numerical Analysis 1981; 18(6):1019-1057.

30. Carey GF, Dinh HT. Grading functions and mesh redistribution. SIAM Journal on Numerical Analysis October 1985; 22(5):1028-1040.

31. Delfour M, Payre G, Zolesio JP. An optimal triangulation for second-order elliptic problems. Computer Methods in Applied Mechanics and Engineering 1985; 50:231-261.

32. Oden JT, Strouboulis T, Devloo P. Adaptive finite element methods for the analysis of inviscid compressible flow. Part 1: Fast refinement/unrefinement and moving mesh methods for unstructured meshes. Computer Methods in Applied Mechanics and Engineering 1986; 59:327-362.

33. Hawken DF, Gottlieb JJ, Hansen JS. Review of some adaptive node-movement techniques in finite-element and finite-difference solutions of partial differential equations. Journal of Computational Physics 1991; 95:254-302.

34. Onate E, Bugeda G. A study of mesh optimality criteria in adaptive finite element analysis. Engineering Computations 1993; 10:307-321.

35. Viswanadham K, Koneru SR. Finite element method for one-dimensional and two-dimensional time dependent problems with B-splines. Computer Methods in Applied-Mechanics and Engineering 1993; 108:201-222.

36. Baines MJ. Algorithms for optimal discontinuous piecewise linear and constant $L_{2}$ fits to continuous functions with adjustable nodes in one and two dimensions. Mathematics of Computation 1994; 62(206):645-669.

37. Baines MJ. On the relationship between the moving finite-element procedure and best piecewise $L_{2}$ fits with adjustable nodes. Numerical Methods for Partial Differential Equations 1994; 10:191-203.

38. Chen KE. Error equidistribution and mesh adaptation. SIAM Journal on Scientific Computing 1994; 15(4): $798-818$.

39. Huang W, Ren Y, Russel RD. Moving mesh partial differential equations (MMPDEs) based on the equidistribution principle. SIAM Journal on Numerical Analysis 1994; 31(3):709-730.

40. Huang W, Sloan DM. A simple adaptive grid method in two dimensions. SIAM Journal on Scientific Computing 1994; 15(4):776-797.

41. Li LY, Bettess P. Notes on mesh optimal criteria in adaptive finite element computations. Communications in Numerical Methods in Engineering 1995; 11:911-915.

42. Kantorovitch LV. Bulletin of the Academy of Sciences of U.S.S.R., 1903; (5).

43. Kantorovitch LV. Applied Mathematics and Mechanics 1942; 6:31-40.

44. Giannacopoulos D. Optimal discretization-based adaptive finite element analysis for electromagnetics. Ph.D. Thesis, McGill University, Montreal, 1998. 
45. Silvester P. Tetrahedral polynomial finite elements for the Helmholtz equations. International Journal for Numerical Methods in Engineering 1972; 4:405-413.

46. Ramo S, Whinnery JR, Van Duzer T. Fields and Waves in Communication Electronics (3rd edn), Wiley: New York, 1993.

47. Fox C. An Introduction to the Calculus of Variations. Oxford University Press: Oxford, England, 1963.

48. Babuska I, Rodriguez R. The problem of the selection of an a posteriori error indicator based on smoothening techniques. International Journal for Numerical Methods in Engineering 1993; 36:539-567.

49. Giannacopoulos D, McFee S. An experimental study of superconvergence phenomena in finite element magnetics. IEEE Transactions on Magnetics 1997; 33(5):4137-4139.

50. Van Bladel J. Singular Electromagnetic Fields and Sources. Oxford University Press: New York, 1991.

51. McFee S, Webb JP. Adaptive finite element analysis of microwave and optical devices using hierarchal triangles. IEEE Transactions on Magnetics 1992; 28(2):1708-1711.

52. Dennis Jr JE, Schnabel RB. Numerical Methods for Unconstrained Optimization and Nonlinear Equations. Prentice-Hall: Englewood Cliffs, NJ, 1983.

53. Webb JP, McFee S. The use of hierarchal triangles in finite element analysis of microwave and optical devices. IEEE Transactions on Magnetics 1991; 27(5):4040-4043.

54. McFee S, Webb JP. Automatic mesh generation for $h-p$ adaption. IEEE Transactions on Magnetics 1993; 29(2):1894-1897.

55. McFee S, Giannacopoulos D. The implications of parallel processing on $h-p$ adaptive finite element analysis for electromagnetics. IEEE Transactions on Magnetics 1998; 34(5):3284-3287.

56. Webb JP, McFee S. The use of hierarchal triangles in finite-element analysis of microwave and optical devices. IEEE Transactions on Magnetics 1991; 27(5):4040-4043.

57. Golias NA, Tsiboukis TD. Adaptive refinement strategies in 3D. IEEE Transactions on Magnetics 1993; 29(2):1886-1889.

58. Janicke L, Kost A. Error estimation and adaptive mesh generation in the 2D and 3D finite element method. IEEE Transactions on Magnetics 1996; 32(3):1334-1337.

59. Kallinderis Y, Vijayan P. Adaptive refinement-coarsening scheme for three-dimensional unstructured meshes. American Institute of Aeronautics and Astronautics Journal 1993; 31(8):1441-1447.

60. Jack AG, Finch JW, Wright JP. Adaptive mesh generation applied to switched-reluctance motor design. IEEE Transactions on Industry Applications 1992; 28(2):370-375. 